\title{
Jeeg: temporal constraints for the synchronization of concurrent objects
}

\author{
Giuseppe Milicia ${ }^{1, *, \dagger}$ and Vladimiro Sassone ${ }^{2}$ \\ ${ }^{1}$ Basic Research in Computer Science (BRICS), University of Aarhus, \\ IT-parken Aabogade 34, DK-8200 Aarhus, Denmark \\ ${ }^{2}$ Informatics, University of Sussex, Brighton BN1 9QH, U.K.
}

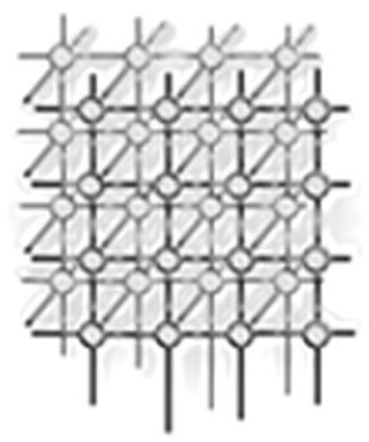

\section{SUMMARY}

We introduce Jeeg, a dialect of Java based on a declarative replacement of the synchronization mechanisms of Java that results in a complete decoupling of the 'business' and the 'synchronization' code of classes. Synchronization constraints in Jeeg are expressed in a linear temporal logic, which allows one to effectively limit the occurrence of the inheritance anomaly that commonly affects concurrent objectoriented languages. Jeeg is inspired by the current trend in aspect-oriented languages. In a Jeeg program the sequential and concurrent aspects of object behaviors are decoupled: specified separately by the programmer, these are then weaved together by the Jeeg compiler. Copyright (c) 2005 John Wiley \& Sons, Ltd.

KEY WORDS: Java; inheritance anomaly; temporal logic

\section{INTRODUCTION}

In the late 1980s, the first experiments in mixing object-oriented programming languages and concurrency unveiled serious difficulties in merging the two concepts [1,2]. Typically, the code for concurrency control, interwoven in the business code of classes, represented an obstacle to code inheritance, making it essentially impossible even in simple, common situations. The term inheritance anomaly [3] was coined to refer to this issue. Indeed, the problems arising from the interaction of inheritance and concurrency were considered so severe as to suggest removing inheritance from concurrent object-oriented languages entirely [1].

Commonly, in object-oriented code, the set of messages accepted by an object is not uniform in time. Depending on the object's state, some of its methods will be unavailable such as, for example,

*Correspondence to: Giuseppe Milicia, Basic Research in Computer Science (BRICS), University of Aarhus, IT-parken Aabogade 34, DK-8200 Aarhus, Denmark.

${ }^{\dagger}$ E-mail: milicia@brics.dk 
pop from an empty stack or put on a full buffer. In sequential situations, it is sometimes conceivable for clients to keep track of which methods are enabled and which are not. For instance, it could be required of the stack user to know at any given point in time whether or not the stack is empty. In a concurrent scenario, however, this is clearly not an option. Clients have no way of knowing about other clients, and any cooperation in this respect requires non-trivial, specific protocols. Our only option is to interweave the stack code with code that controls access from clients. Concurrent objects must take direct control of their synchronization code, and the phenomenon of inheritance anomaly sets in, forcing programmers to override inherited code in order to refine the synchronization code therein. The situation can be exemplified in a simple case by the following idealized pseudo-code of a buffer:

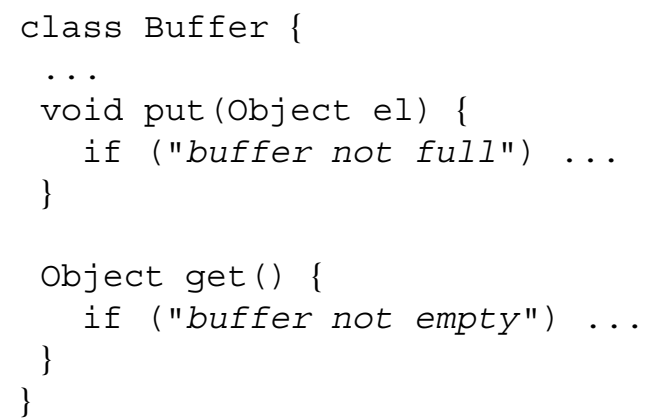

Suppose now that to enhance Buffer we wish to add, for instance, the method freeze that makes it read-only. Whatever the original chunks of code for "buffer not . . ", chances are that they must be totally rewritten to take into account the new enabling condition.

Generally speaking, the inheritance anomaly has been classified in three broad varieties [3] that we review below.

Partitioning of states. Inspired by the example above, one may disentangle code and synchronization conditions by describing methods' enabling according to a partition of the object's states. To describe the behavior of the class Buffer, for instance, the state can be partitioned in three sets, empty, partial and full, the former containing the states in which the buffer is empty-so that get is inhibited — the latter those in which it is full—so that is put to be disallowed. One can then specify

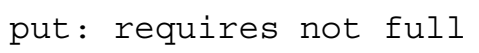

and refine the code of get and put to specify the state transitions. For instance, get would declare the conditions under which the buffer becomes empty or partial:

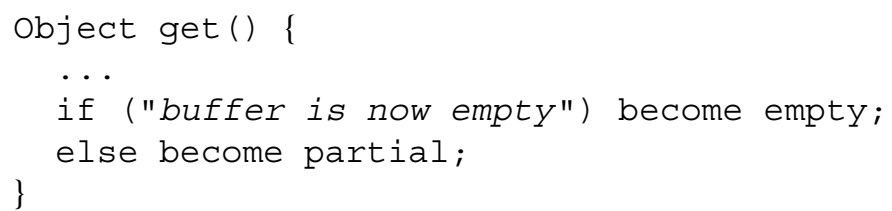

The inheritance anomaly here surfaces again, as derived classes may force a refinement of the state partition. As an example, consider adding a method get 2 that retrieves two elements at once. 
Alongside empty and full, it is necessary to distinguish those states where the buffer contains exactly one element. Clearly, the state transitions specified in get and put must be re-described accordingly.

History-sensitiveness of acceptable states. When method enabling depends on its past history rather than depending on the object's state, as above, a different form of inheritance anomaly occurs. Suppose for instance that we want to refine our buffer with a method gget that works like get but that cannot be executed immediately after a get. Clearly, that can only be achieved in Java by adding code to get to keep track of its invocations. That is, we have to rewrite the entire class. We revisit this problem later on.

Modification of acceptable states. A third kind of anomaly happens with mix-in classes, that is classes created to be mixed-into other classes to add to their behavior. The typical situation arises when one wishes to enrich a class with a method that influences the acceptance states of the original class' methods. Our previous example of the method freeze belongs essentially to this category of anomaly. Similarly, it is reasonable to expect to be able to design a

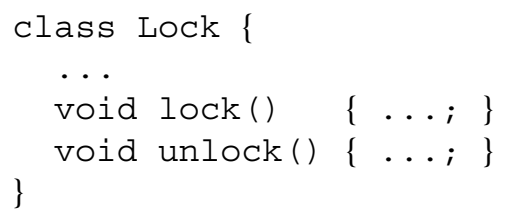

to be used to add lock capabilities to clients classes by means of the standard inheritance mechanism. However, clearly enough, (multiple) inheritance of Lock and Buffer does nothing towards creating a lockable buffer, unless we completely recode get and put to keep into account the state of the Lock component of the object.

Although modern programming languages provide concurrency and inheritance, the inheritance anomaly is most commonly ignored. Indeed, Java and C\# are mainstream concurrent object-oriented languages whose synchronization primitives are based exclusively on (a non-declarative use of) locks and monitors.

Although no generally accepted solution has emerged so far, several approaches have appeared in the literature that mitigate the inheritance anomaly. Our proposal, Jeeg, focuses on Java. Jeeg is a dialect of Java based on method guards whose particularity is to address history-sensitive inheritance anomaly. As in guard-based languages, methods are labeled by formulae that describe their enabling condition. The novelty of the approach is that we use (a version of) Linear Temporal Logic (LTL) [4], so as to allow the expression of properties based on the history of the computation. Exploiting the expressiveness of LTL, Jeeg is able to single out situations such as those described in the examples above, thus ridding the language of the corresponding anomalies. Due to the nature of the problem, it is of course impossible to claim formally that a language avoids the inheritance anomaly or solves it. The matter depends on the synchronization primitives of the language of choice, and new practices in object-oriented programming may at any time unveil shortcomings unnoticed before, leading to new kinds of anomalies. Nevertheless, since the expressive power of LTL is clearly understood, one of the pleasant features of Jeeg is that it comes equipped with a precise characterization of the situations it can address. More precisely, we will see that all the anomalies depending on sensitivity to object histories that are expressible as star-free regular languages can, in principle, be avoided in Jeeg. 
The current implementation of Jeeg relies on the large body of theoretical work on LTL, which provides powerful model-checking algorithms and techniques. Currently, each method invocation incurs an overhead that is linear in the size of the guards appearing in the method's class. Also, the evaluation of the guards at runtime requires mutual exclusion guarantees that have a (marginal) computational cost. When compared with the benefit of a substantially increased applicability of inheritance, we feel that this is a mild price to pay, especially in the common practical situations where code overriding is infeasible or cost-ineffective. At the same time, we are working on alternative ways to implement the ideas of Jeeg, aiming both at a lower computational overhead and at more expressive logics.

Jeeg is an aspect-oriented language. Synchronization constraints, expressed declaratively, are totally decoupled from the body of the method, so as to enhance separation of concerns. The structure of the paper is as follows: Section 2 presents the language, while Section 3 cures the classical inheritance anomalies with it; Section 4 treats the expressive power of Jeeg. More details on the language and its current implementation are provided, respectively, in Sections 5 and 6. In Section 7 we discuss the performance overhead brought forth by the Jeeg methodology. Finally, we discuss related and further work. The appendices provide some optional material, most notably an example of Jeeg-toJava translation.

\section{A TASTER OF JEEG}

Jeeg differs from Java in the use of new synchronization primitives which replace the wait (), notify (), and notifyAll ( ) constructs. In Jeeg the synchronization code of a class is not inlined in its methods; rather it is specified separately. This can be done either via a sync section of the class definition or via an XML file associated with the class. In the former case, a Jeeg class has the following structure:

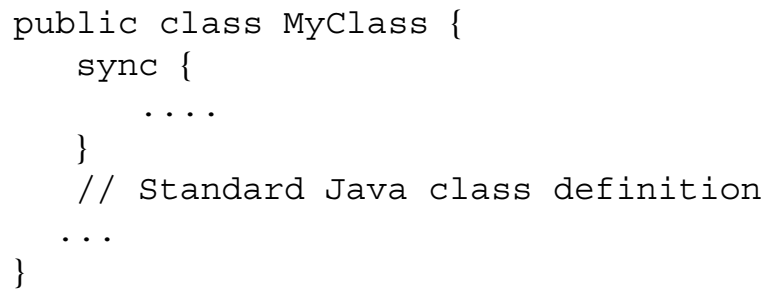

The sync section consists of a sequence of declarations of the form:

$\mathrm{m}: \phi ;$

where $\mathrm{m}$ is a method identifier and $\phi$, the guard, is a formula in a given constraint language to be described shortly. Methods associated with a guard are said guarded. Intuitively, $\mathrm{m}: \phi$ means that at a given point in time a method invocation $\circ . \mathrm{m}()$ can be executed if and only if the guard $\phi$ evaluated on object $\circ$ yields true. Otherwise, the execution of $\mathrm{m}$ is blocked until $\phi$ becomes true. Then, the resumption of $m$ follows the familiar rules of the Java notifyAll primitive. Guarded methods are executed in mutual exclusion at the level of objects. Indeed, from a Java perspective, every guarded 
method is implicitly synchronized. Synchronization constraints in Jeeg are thus exclusively at the method level: there is no synchronized key word and it is not possible to define guarded regions.

The XML description of synchronization complies with the document type definition (DTD) of Appendix A and is described later in Section 5.

The expressive power of this model of synchronization depends of course on the choice of the constraint language. Indeed, if we limit $\phi$ to Java Boolean expressions we obtain a declarative version of the standard synchronization mechanism of Java.

\subsection{The constraint language}

Choosing the constraint logic is a trade-off between expressiveness and efficiency, as the truth of formulae must be verified at every method invocation. We need a logic that is more expressive than Java Boolean expressions but does not substantially worsen the computational cost of formula evaluation, so that the computational overhead does not overcome the expressiveness benefits. A logic that suits our purpose is LTL [4]. As we shall see, (a variation of) LTL used in the context of Jeeg gives a substantial improvement in the expressiveness of Java Boolean expressions, allowing in particular the vanishing of the history-sensitive inheritance anomaly, and at the same time keeps the overhead on evaluation time on the linear scale.

LTL introduces time in propositional and first-order logic. It becomes possible to reason about dynamic, evolving systems by expressing properties referring to what happened in the past or to what will happen in the future. For example, one can write

$$
\text { Previous } x>0
$$

which holds if those system states whose preceding state validates the proposition ' $x$ is greater than 0 ', or also

$$
x>0 \text { Since } y<0 \text {, }
$$

true if at some point in time $y$ was less than 0 and at all subsequent instants (that is since then) $x$ has been positive.

The syntax of our constraint language of choice, $\mathrm{CL}$, is as follows:

$$
\phi::=\operatorname{AP}|! \phi| \phi \& \& \phi|\phi||\phi| \text { Previous } \phi \mid \phi \text { Since } \phi
$$

A formula $\phi$ of $\mathrm{CL}$ is defined starting from atomic formulas $\mathrm{AP}$, denoted by $p, q, \ldots$, which are Java Boolean expressions. We consider exclusively pure Boolean expressions, with no side-effects, method invocations or references to objects (other than the implicit references to self); also, $\phi$ can only refer to private/protected fields of the class it belongs to. Note that we could allow particular methods which can be assumed to have no side-effects, e.g. Object.equal (), in an ad hoc manner. CL has the obvious conjunction $\& \&$, disjunction || and negation ! connectives. In addition to these, it provides two temporal past operators: previous and since, whose informal meaning we described above. This logic is a variation of LTL known as past tense LTL [5]. By combining the basic operators it is possible to define two interesting, self-explanatory, auxiliary ones: always, sometime. Formally, Sometime $\phi \triangleq$ true Since $\phi$ and Always $\phi \triangleq$ !Sometime ! $\phi$. For the user's convenience, these operators are predefined in the Jeeg implementation. 
All this would not be very helpful in our attempt to tackle the history-sensitive anomaly without a way to refer to the history of object method invocation. The notion of event introduced below serves this purpose.

Definition. (Event) An event for object $o$ is the execution of one of its methods.

From this basic notion we can define $\mathcal{H}_{\pi}(o)$, the history of object $o$ in (a concurrent) computation $\pi$. Informally, this is the sequence of the events of $o$ in $\pi$, in the order they occur, together with the states they connect. In order to make this precise, observe that thanks to our assumption that guarded methods run in mutual exclusion, each computation unambiguously defines a sequence of method invocations for each object involved. So, without loss of generality, as far as $o$ is concerned, the generic computation $\pi$ will have the shape

$$
h_{0}^{0} \cdots h_{j_{0}}^{0} o . \mathrm{m}_{1} h_{0}^{1} \cdots h_{j_{1}}^{1} o . \mathrm{m}_{2} h_{0}^{2} \cdots h_{j_{2}}^{2} \cdots
$$

where $\mathrm{m}_{i}$ are all the activations of a guarded method of $o$ in $\pi$ and $h_{0}^{i} \cdots h_{j_{i}}^{i}$ are sequences of Java heaps (such sequences arise by assignments to public variables or method invocations-either of the unguarded and other objects' methods). Formally, $\mathcal{H}_{\pi}(o)$ can then be defined by induction on $k$, the number of $o$ guarded methods in $\pi$,

$$
\mathcal{H}_{\pi}(o)= \begin{cases}h_{j_{0}}^{0} & \text { for } k=0 \\ \mathcal{H}_{\pi_{k}}(o) \mathrm{m}_{k} h_{j_{k}}^{k} & \text { for } k>0\end{cases}
$$

where $\pi_{k}$ is the subcomputation of $\pi$ terminating just before the invocation on $o . m_{k}$.

Note that such a definition makes perfect sense under our hypothesis. As guards may only refer to private/protected variables, their value can only be affected by invocation of methods of $o$. It is therefore a sensible choice to assume $h_{j_{0}}^{0} h_{j_{1}}^{1} h_{j_{2}}^{2} \cdots$ as the sequence of states of $o$ for the evaluation of temporal guards. Note also that only the part of $h_{j k}^{k}$ containing the values of non-reference private/protected variables of $o$, say $\sigma_{k}$, is needed for that. We therefore represent object histories by sequences such as

$$
\mathcal{H}_{\pi}(o) \equiv \sigma_{0} \stackrel{m_{1}}{\longrightarrow} \sigma_{1} \stackrel{m_{2}}{\longrightarrow} \sigma_{2} \stackrel{m_{3}}{\longrightarrow} \sigma_{3} \cdots
$$

where the computation $\pi$ will most often remain implicit. To exemplify the definition consider the simple counter class in Figure 1.

If we execute

Counter $\mathrm{C}=$ new Counter ();

c.inc ();

c.inc ();

c. $\operatorname{dec}() ;$

we obtain the history in Figure 2. Interwoven executions in the presence of concurrent objects easily become more complex. Nevertheless, the notion of history of each single object remains relatively simple. Figure 3, for instance, illustrates histories in the case of two concurrent threads executing the code above on two distinct counters.

For practical convenience we will think of event $\mathrm{m}_{i}$ as a reference to a special identifier event in $\sigma_{i}$. So, we will write

$$
\mathcal{H}(o) \equiv \sigma_{0} \sigma_{1} \sigma_{2} \sigma_{3} \cdots
$$




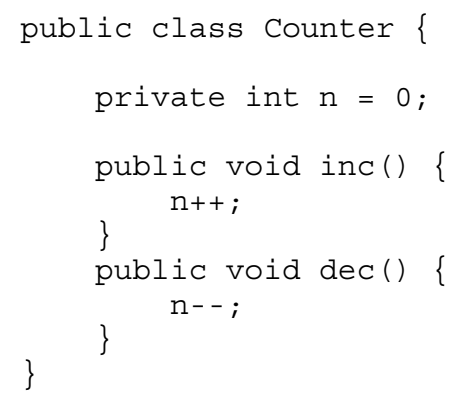

Figure 1. A simple counter.

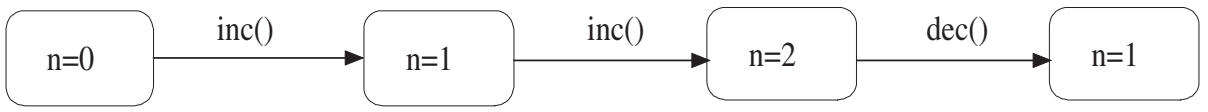

Figure 2. History

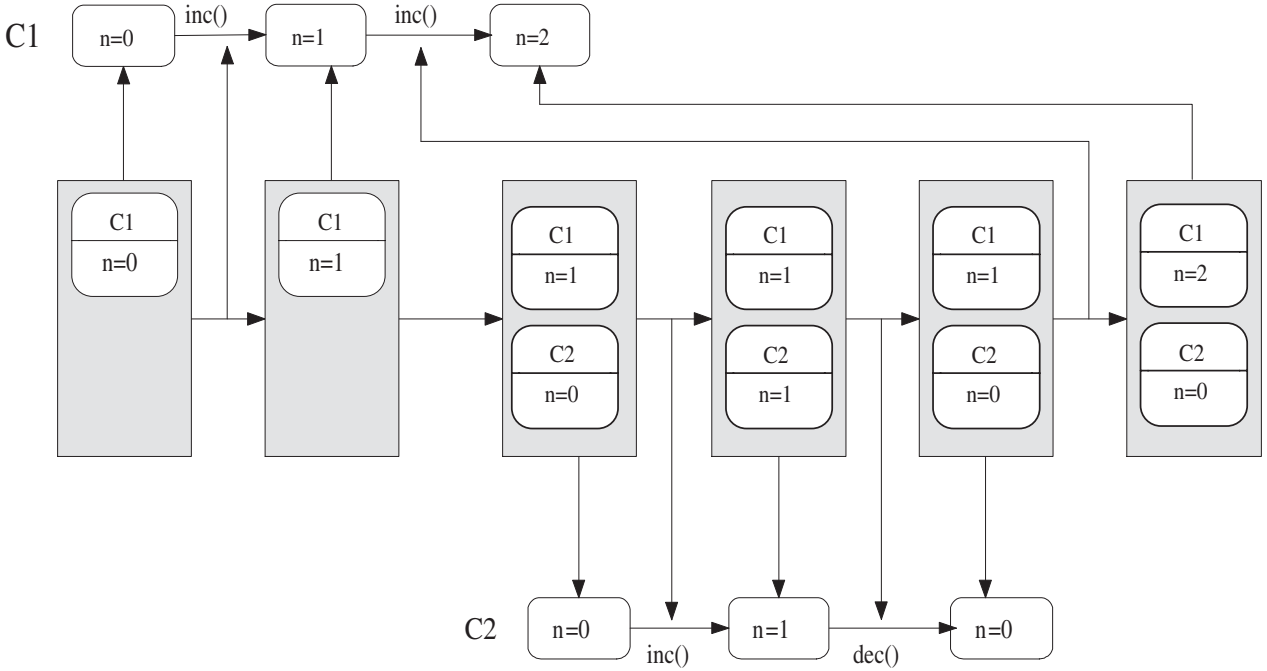

Figure 3. Extracting the history. 


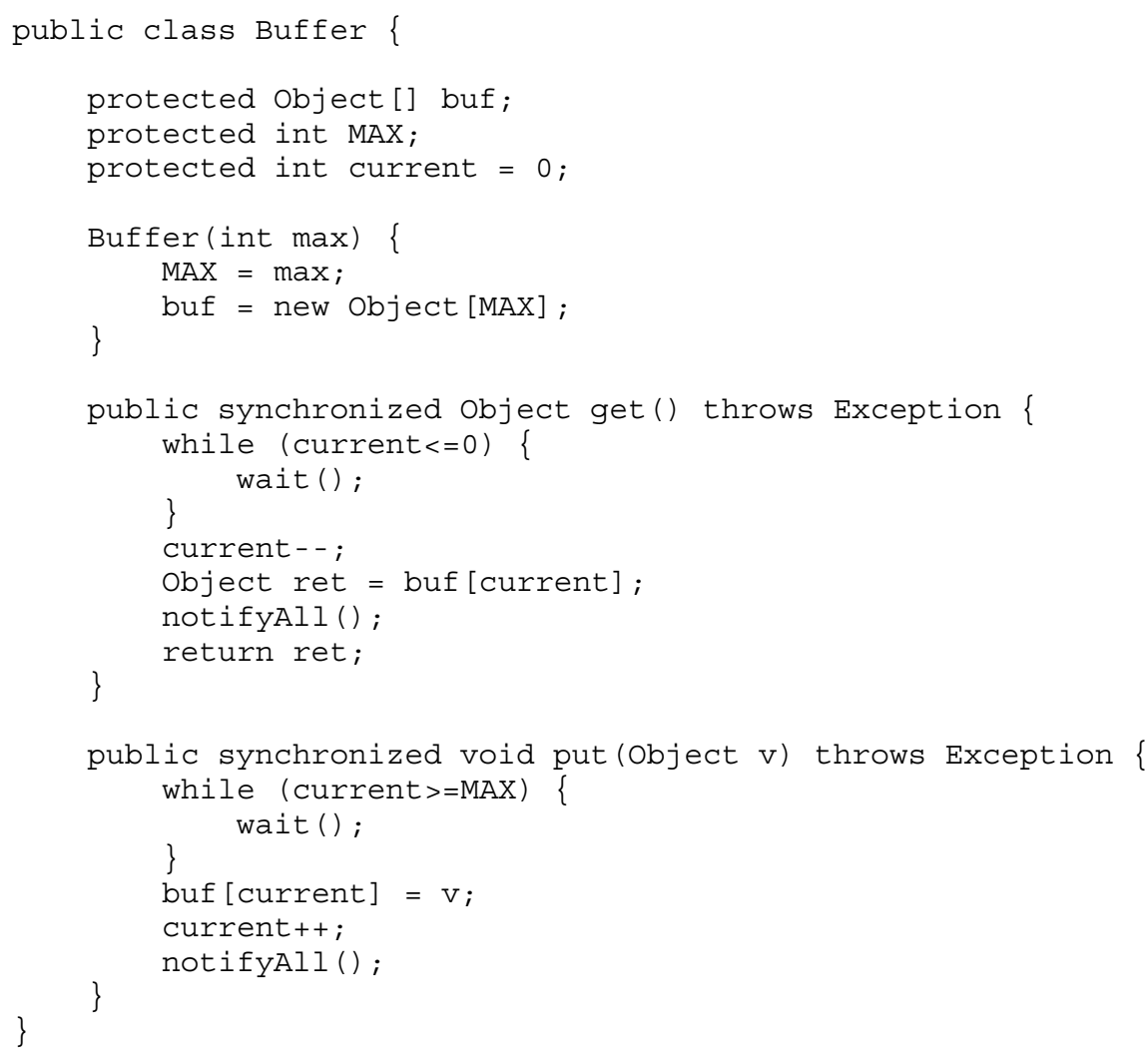

Figure 4. Concurrent bounded buffer in Java.

with the understanding that $\sigma_{i}$ binds the identifier event to (a value representing method) $\mathrm{m}_{i}$. (References to event are undefined in $\sigma_{0}$.) For example, in the third state in Figure 2, event yields inc. Identifier event can be used by CL. In this way history information finds its way into our constraint language.

Next, we give formal semantics to CL by defining the relation $\mathcal{H}_{\pi}(o) \models \phi$ expressing that property $\phi$ holds for object $o$ after a computation $\pi$. Let $\Sigma$ denote $\mathcal{H}_{\pi}(o)$. For all indexes $k$ in $\Sigma$, we define $\Sigma_{k} \models \phi$, that is $\phi$ holds at time $k$, by structural induction on $\phi$ as follows:

$$
\begin{array}{rll}
\Sigma_{k} \models p & \text { iff } & \sigma_{k} \models p \quad\left(p \text { is true at } \sigma_{k}\right) \\
\Sigma_{k} \models ! \phi & \text { iff } & \text { not } \Sigma_{k} \models \phi \\
\Sigma_{k} \models \phi \| \psi & \text { iff } & \Sigma_{k} \models \phi \text { or } \Sigma_{k}=\psi \\
\Sigma_{k}=\text { Previous } \phi & \text { iff } & k>0 \text { and } \Sigma_{k-1} \models \phi \\
\Sigma_{k} \models \phi \text { Since } \psi & \text { iff } & \Sigma_{j} \models \psi \text { for some } j \leq k,
\end{array}
$$

Finally, we convene that $\Sigma \models \phi$ iff $\Sigma_{0} \models \phi$. 


\section{THE INHERITANCE ANOMALY}

A striking example of the inheritance anomaly, borrowed from [3] and already mentioned in the introduction, applies to the class Buffer in Figure 4, a simple implementation of a bounded buffer in Java. Consider defining a subclass of Buffer that provides an additional method gget that removes an element from the buffer only if the last operation performed by the buffer was not a get. The class HistoryBuffer in Figure 5 is a possible solution. It illustrates a characteristic occurrence of the inheritance anomaly. Ideally, we would expect method gget to be independent of the methods defined in the parent class. A deeper analysis shows that gget can only be implemented if both inherited are redefined, resulting in the loss of any code reuse that inheritance should have provided.

The example in Figure 5 follows closely the original presentation seen in [3]. However, it is possible to minimize the amount of code rewriting relying on the implementation of the methods get and put found in the super-class. We could write

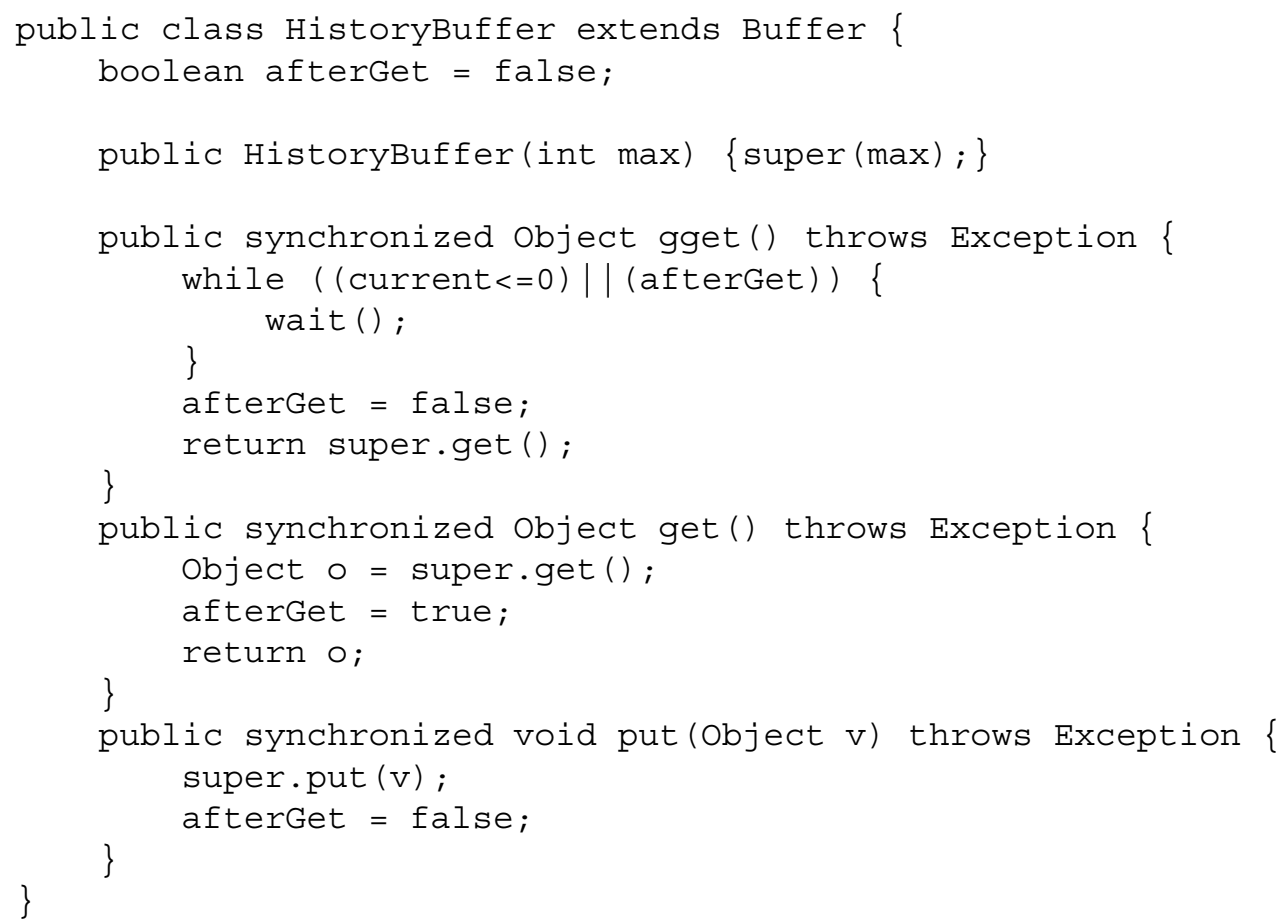

This comes at the price of some redundant synchronization. Nevertheless, the problem remains. The addition of the method gget forces us to revise the implementation of seemingly unrelated inherited methods.

This kind of anomaly arises from the fact that gget is a history-sensitive method. Generally speaking, the inheritance anomaly depends on the synchronization primitives present in the language, and different primitives result in different varieties of anomaly [3]. In particular, languages based on method guards and their cousin technologies run the risk of suffering from the history-sensitiveness of acceptable states. This is indeed the case of Jeeg, as its synchronization mechanisms are based on 


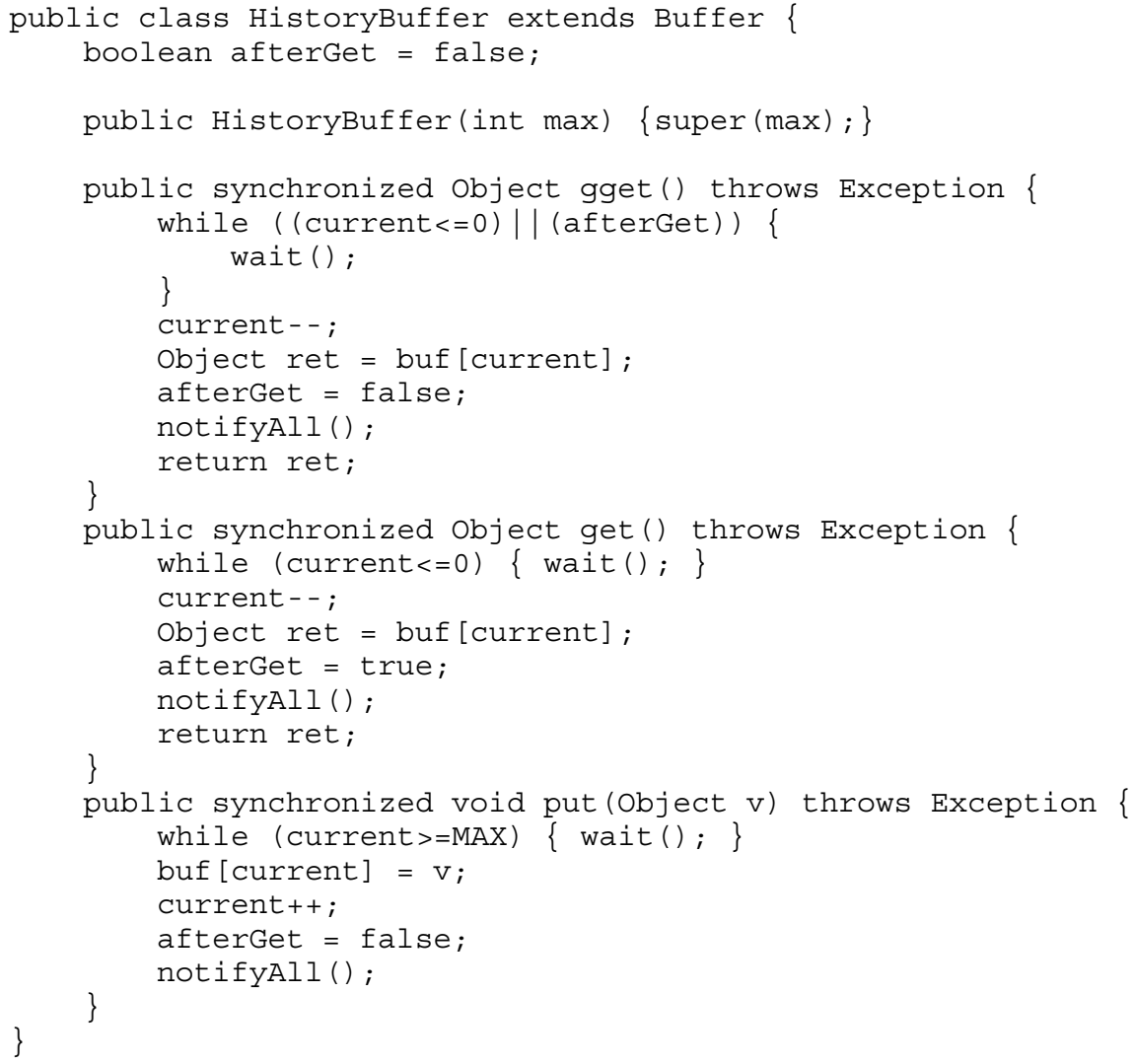

Figure 5. The class HistoryBuffer in Java.

a variation of method guards. Therefore, a good test of expressiveness for Jeeg is given by handling subclassing by history sensitive methods and gget above. It should not come as a surprise now that the additional expressive power added to method guards by the temporal aspects of CL suffices to solve several occurrences of the inheritance anomaly. In this section we exemplify such expressiveness, while in the following we try to quantify it formally.

Consider the Jeeg version of the class Buffer as defined in Figure 6. We can define a class HistoryBuffer in Jeeg as in Figure 7. This example shows how the use of the temporal operator Previous avoided the occurrence of the inheritance anomaly. We no longer need to introduce an instance variable to keep track of the last operation performed. CL gives us enough expressive power to do without.

As already discussed in the introduction, a different kind of inheritance anomaly that plagues guardbased languages arises in the case of mix-in classes. In [3], the authors use multiple inheritance to 


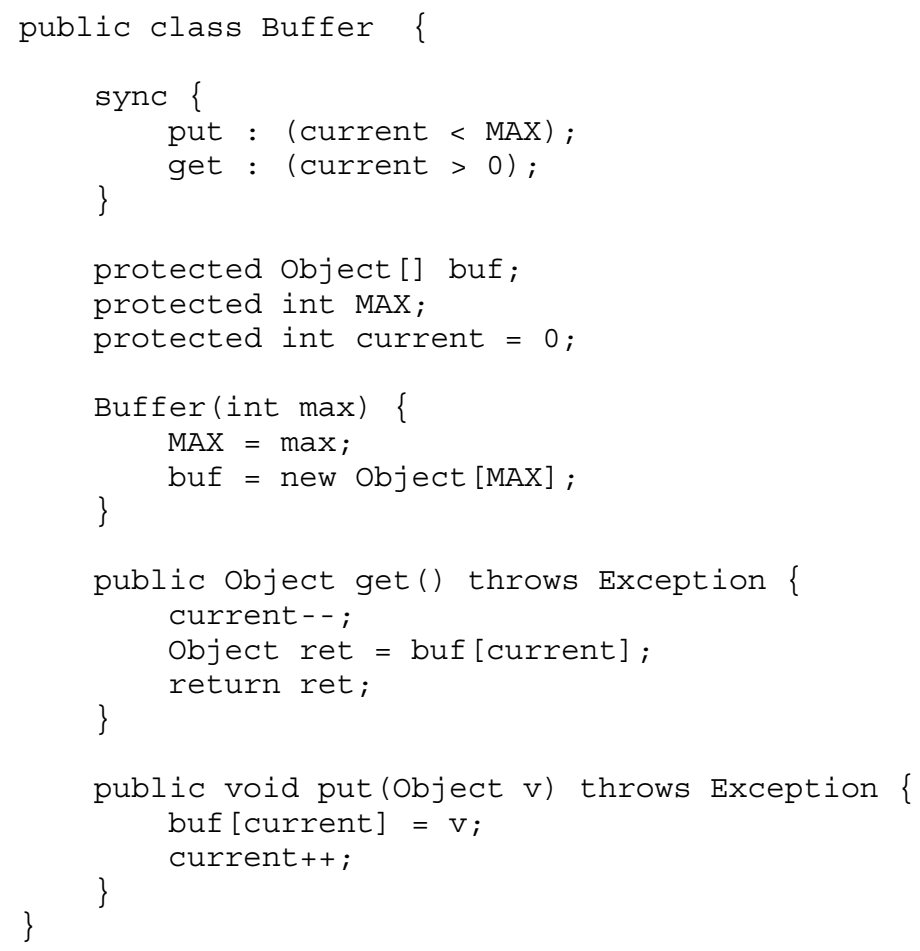

Figure 6. The Buffer class in Jeeg.

show this variant of the inheritance anomaly. Java and Jeeg do not provide multiple inheritance, but the use of interfaces results in similar problems. Consider the class LockBuf in Figure 8. This is a subclass of the class Buffer that implements the Lock interface resulting in a lockable buffer. A locked buffer must not accept any other message than unlock. One would expect the newly introduced methods to be orthogonal to the inherited ones (this would seem even more natural if they were inherited by multiple inheritance). Naturally, in Java, we cannot simply implement the Lock interface to have a lockable buffer, as methods put and get need to be redefined to account for the new locked and unlocked states, possibly introducing a new Boolean variable locked to distinguish between the two states the buffer can be in. Jeeg solves the problem elegantly, as can be seen in Figure 8, again by exploiting the temporal operators of the constraint language. Indeed, lock and unlock are history-sensitive methods. Note that the synchronization constraints of the inherited methods are overridden, while the method definitions are not. As explained in Section 5 below, in Jeeg method definitions and their synchronization constraints are orthogonal and can be overridden/inherited separately. As expected, the syntax super. getConstr allows us to refer to the 


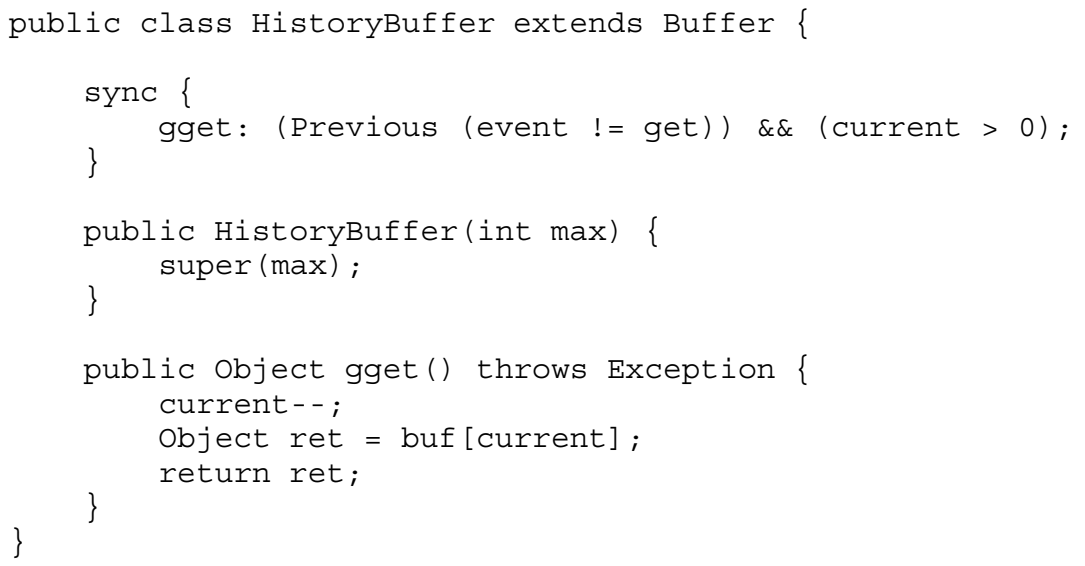

Figure 7. The HistoryBuffer class in Jeeg.

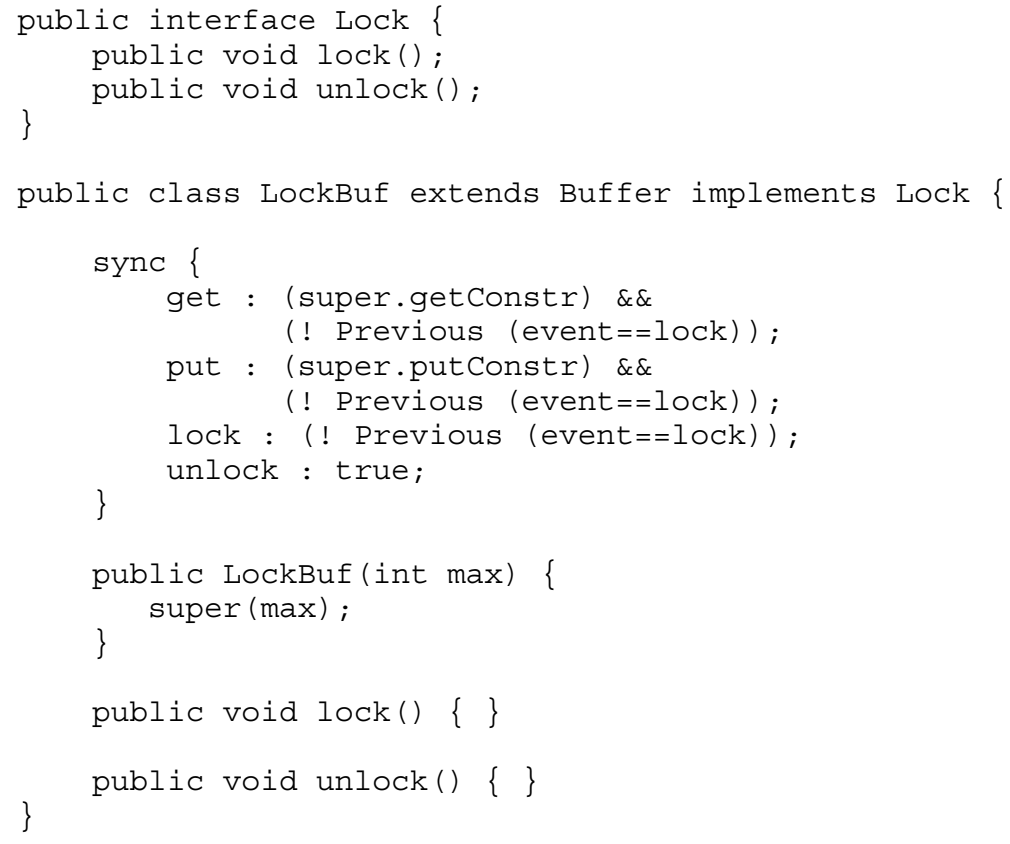

Figure 8. A lockable buffer. 
synchronization constraint of a given method, get in this case, as defined in the super class. In general, for the constraint attached to method $m$ in the super class, we write super.mCostr.

\section{EXPRESSIVENESS OF JEEG}

When introducing a new synchronization primitive in a concurrent object-oriented language, it is often difficult to assess its impact on the inheritance anomaly in a quantitative manner. Building on the large body of results on LTL, such analysis is however possible for Jeeg. In particular, we adapt to our context a characterization of LTL expressiveness in term of 'star-free' regular languages. (For a thorough introduction to LTL the reader is referred to [6].)

The question we are interested in is to what degree does Jeeg solve the inheritance anomaly? According to [3], in a language like Java the anomaly arises when the observable behavior of an object is more complex than what can be ascertained from its internal state. For instance, the internal state of a Buffer object cannot account for the information of whether or not the last method to be executed was a get. Therefore, in order to define gget, we need to refine the internal state of the object, which comes at a heavy price. The constraint language of Jeeg, however, allows us to describe sequences of events and so ascertain more behaviors from the same state. As long as CL can describe a certain sequence, we can write a constraint that avoids the need of state refinement. A measure of how much of the inheritance anomaly disappears in Jeeg can thus be obtained by measuring which sequences of states are definable in CL. For the purpose of this section, we assume AP finite.

Definition. (General Regular Expressions) Given a finite alphabet $A$, the regular expressions over $A \cup\{\epsilon\}$, where $\epsilon$ is a special symbol such that $\epsilon \notin A$, are defined by the following grammar:

$$
r e::=\epsilon|a| r e \cdot r e|r e+r e| \neg r \mid r e *
$$

where $\epsilon$ denotes the empty word, $a \in A$ denotes the language consisting of a single string $a$, and •, ,$+ \neg$ and $*$ represent language concatenation, union, negation with respect to $A^{*}$ and Kleene closure, respectively. The star-free regular expressions are the regular expressions with no occurrence of $*$.

A classical result about LTL states that the sets of state sequences definable by LTL formulae on atomic propositions AP coincide with the star-free regular languages on the alphabet $\wp(A P)$, the powerset of AP. Spelling this out, a set of state sequences $X$ is the set of all $\Sigma$ that satisfy a given $\phi$ of LTL if and only if $X$ is a star-free regular language. The reader is referred to [7] for the details.

Applied to our framework, this result gives a first answer to our question: CL can define the sets of sequences of states that are star-free regular languages on finite subsets of AP. To refine this statement, let us observe that we can identify a certain state of an object (or better, its part expressible in CL), by a Boolean formula on its (private/protected) field's values. Let $A_{\mathrm{C}}$ be the set of these Boolean expressions. It follows that a certain sequence of states can be identified by a set of formulae $P$ in $A_{\mathrm{C}}$. Note that, in general, $P$ will denote a set of sequences of states; that is, all the sequences such that $\Sigma \models P$ (meaning $\sigma_{i} \models p_{i}$, for every $i$ ). In this context, the following theorem formalizes the correspondence (from the point of view of the class $\mathrm{C}$ ) between sequences of states denoted by $\mathrm{CL}$ formulae and sequences of states corresponding to star-free regular expressions on $A_{\mathrm{C}}$. 
Theorem. (Characterizing CL) Let $C$ be a class and $X$ a set of state sequences. Then, for a given $C L$ formula $\phi$ on $C, X=\{\Sigma \mid \Sigma \models \phi\}$ if and only if there exists a star-free regular expression re on $A_{C}$ such that $\Sigma \in X$ iff $\Sigma \models P$ for some $P \in$ re.

It is interesting to specialize this result when $\mathrm{AP}$ is restricted to conjunctions of atomic formulae of the kind event $==\mathrm{m}$. In such a case, CL expresses properties of sequences of events-as states are only distinguishable in that respect-and captures precisely those sets of sequences of events that are star-free regular languages on the alphabet of method identifiers.

The characterization in terms of regular languages also provides intuition about what cannot be expressed in CL and, therefore, will result in the occurrence of inheritance anomalies. We show an admittedly contrived example below.

Example. Consider a class representing a simple shared resource which can be simultaneously held by multiple clients:

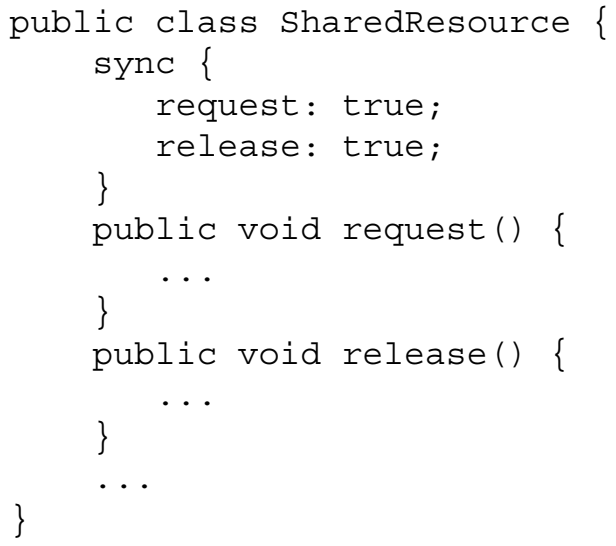

Before using the resource, clients are supposed to call the method request. When the client no longer needs the resource, it should call the method release. To keep the example simple, we assume that clients respect this protocol.

Suppose that we want to define a class SeizableResource that allows clients to gain exclusive access to the shared resource. An additional method exclusiveRequest must be provided. Clearly, this method should be allowed to execute only when no other client is using the resource. To accomplish this we must make sure that any call to the method request was followed by a call to the method release. Unfortunately this constraint cannot be expressed by LTL. Indeed from a language point of view, we want to know whether the history of the object is a word in the language:

$\mathrm{M}::=$ request $\mathrm{M}$ release $|\mathrm{MM}| \epsilon \mid \ldots$

where the dots stand for any method identifier in the class SharedResource. It is well known that this language, a language of balanced parentheses, is not star-free or regular. As a consequence, it is not possible to write a synchronization constraint for the method exclusiveRequest in CL; that is, it is not possible to find a formula that describes the states where exclusiveRequest is enabled. What we need to do is to manually keep track of whether the resource is being used or not: 


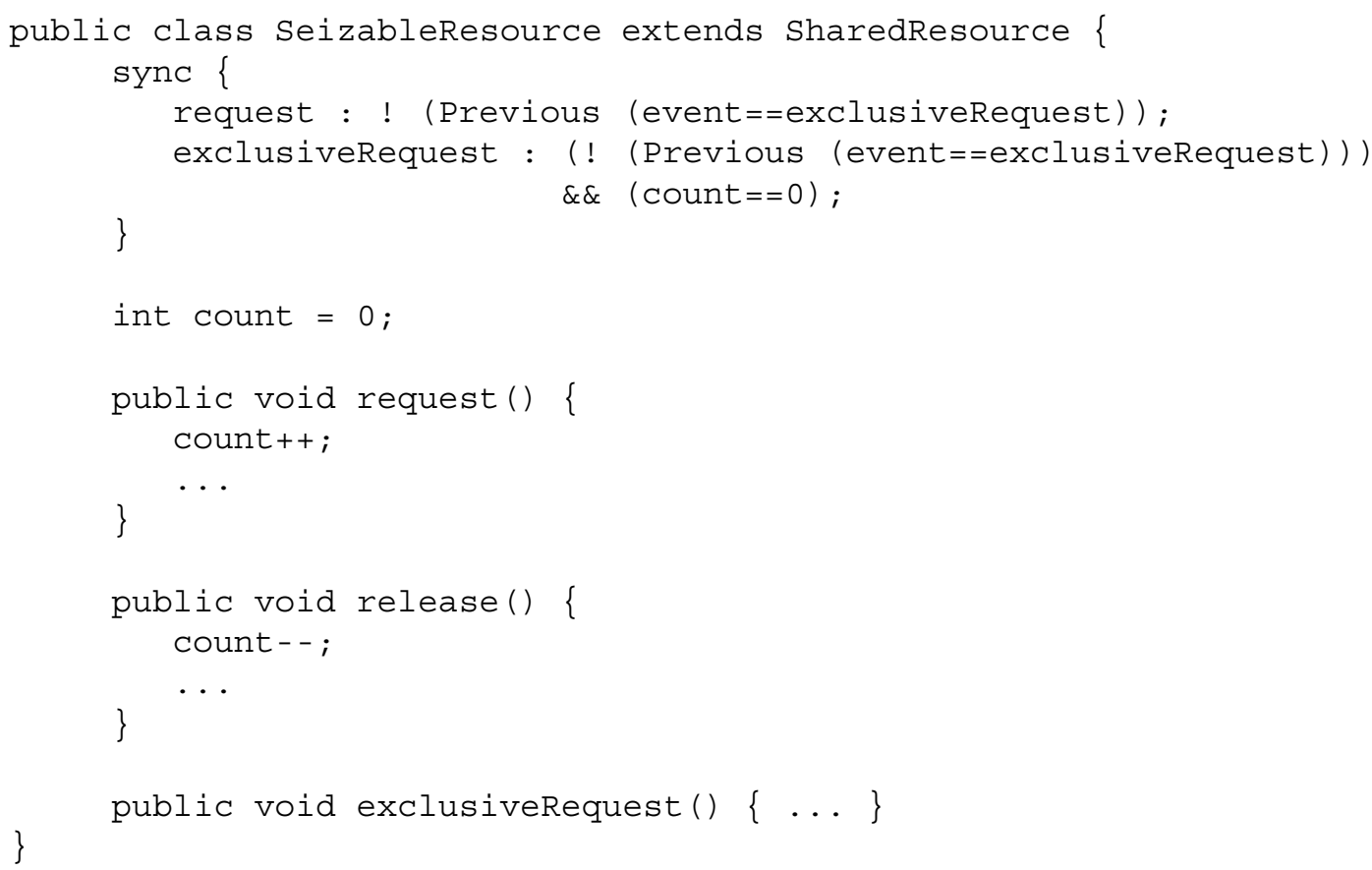

The derived class uses one counter count to ascertain whether the resource is currently being used by any client. To accomplish this book-keeping, it is necessary to redefine the base-class methods request and release.

The example above is typical. A constraint which cannot be expressed in LTL must involve some form of recurrent counting. (For an in-depth discussion on these issues, we again refer the reader to $[6,8]$.)

Example. The classic HistoryBuffer example has been solved using Jeeg in Figure 6. It is interesting to analyze the (simple) temporal constraint used in the example in terms of star-free regular expressions. The constraint relative to the gget method is the following:

(Previous (event $!=$ get)) \&\& (current > 0);

For simplicity let us restrict ourselves to its temporal component:

(Previous (event $!=$ get))

In light of the previous discussion on the equivalence of (past) LTL formulae and star-free regular expressions, we give the same constraint in its regular expression form. Intuitively, the language the formula describes is that of 'all the words in the trace alphabet not ending with the symbol get'. We can define $A^{*} \equiv \epsilon+\neg \epsilon$. With abuse of notation we denote the formula (event = = get) simply as get. In this manner occurrences of the event get in the history of the object could be recorded simply as get. The corresponding (star-free) regular expression is

$$
\neg\left(A^{*} \cdot \text { get }\right)
$$

which formalizes the intuitive set of all the words that do not end with the symbol get. 


\section{DIGGING DEEPER INTO JEEG}

In this section we look deeper into the interaction between Jeeg synchronization primitives and the other available language features.

\section{Synchronized and unsynchronized methods}

In Jeeg, methods for which a synchronization constraint is specified are executed in mutual exclusion. In Java terms, they are synchronized. On the other hand, methods for which no synchronization constraint is specified have no mutual exclusion guarantee. Clearly, an undisciplined use of unsynchronized methods may lead to mutual exclusion problems. This is particularly relevant in our setting as the evaluation of a guard must be atomic in order to be meaningful. If an unsynchronized method attempts to modify an attribute of the object while a guard is being evaluated we may end up with an inconsistent result. A trivial example will clarify the situation.

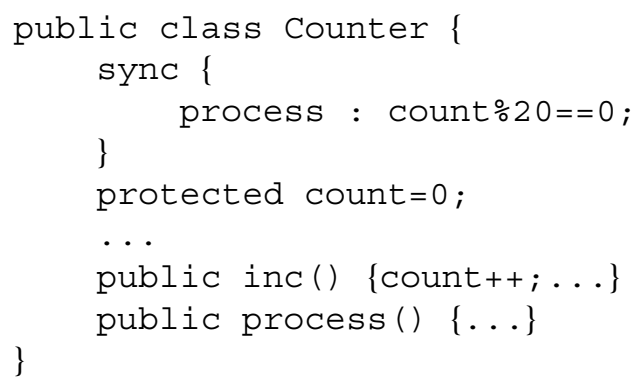

In the example above the method inc is not executed in mutual exclusion, as a consequence it can modify the value of count during a call to the method process and the evaluation of its guard. Naturally, a call to inc can change the value of the guard for process after its evaluation, and this would leave the method process to be executed in an inconsistent state. A similar situation would occur if guards were allowed to use public attributes. To avoid these situations, the attributes occurring in a guard must be accessed in mutual exclusion with the evaluation of the guard. Therefore, in Jeeg attributes used in guards can only be modified by synchronized methods.

Java (and consequently Jeeg) allow methods and attributes to be declared static. Static fields and methods are common to a class rather than to each of its instances. To access a static attribute, therefore, it is not enough to own the lock for a certain object instance. Indeed, such a lock does not guarantee mutually exclusive access to static attributes. The lock needed to obtain such access must be on the class rather than on the object. As a consequence, static fields can only be modified by static synchronized methods. Conversely, static synchronized methods own a lock on the class rather than on a specific object instance. For this reason such methods are forbidden to modify non-static object fields.

Another issue related to unsynchronized methods is that the step-wise history of the object is not well defined as regards to their execution order. Indeed, there can be two methods active at the same time. To force an ordering between unsynchronized methods we adopt the policy of accounting for methods in the history according to the moment their execution finishes. Note, however, that in a multiprocessor system, this notion is not well-defined. It is therefore bad programming practice in 


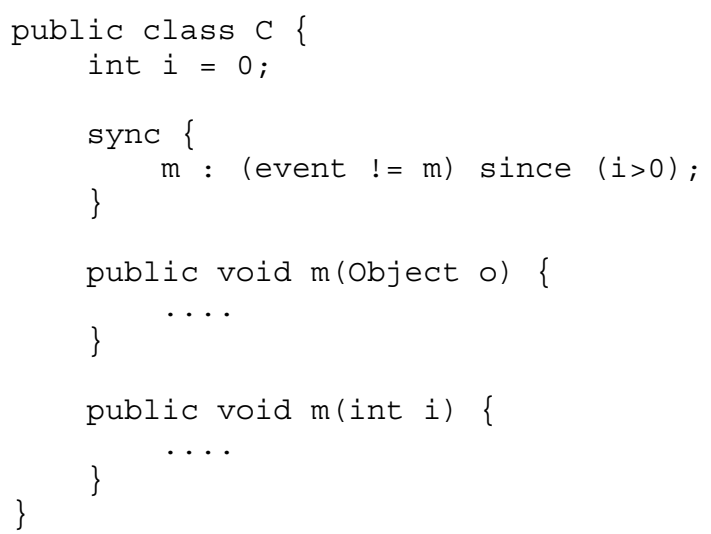

Figure 9. Method overloading.

such systems to rely on guards whose truth values depend on the relative ordering of unsynchronized methods.

\section{Method overloading}

From a synchronization point of view Jeeg does not distinguish between different versions of an overloaded method. The synchronization granularity stops at the method identifier level.

In the example in Figure 9, the synchronization constraint applies to both definitions of the overloaded method $\mathrm{m}$. This choice is motivated by the fact that synchronization constraints relate to the essential behavior of a method, which we feel should not be changed by overloading. One could certainly define an overloaded method $m$ whose definitions access the shared attributes of the object in a completely different manner. It would not be difficult to support these situations by basing synchronization constraints on method signatures rather than identifiers.

\section{Inheritance and method overriding}

Consider a subclass Xbuf of Buffer as defined in Figure 10. There we assume the existence of a support class Couple that only wraps up two values as an object. The new class does not override any method of its base class, therefore the methods are inherited together with their synchronization constraints. The additional constraint for the new method is independent of the existing ones.

In Jeeg method definitions and their synchronization constraints are completely decoupled. This scales up to method overriding and indeed it is possible to selectively override the method definition, its synchronization constraint, or both. 


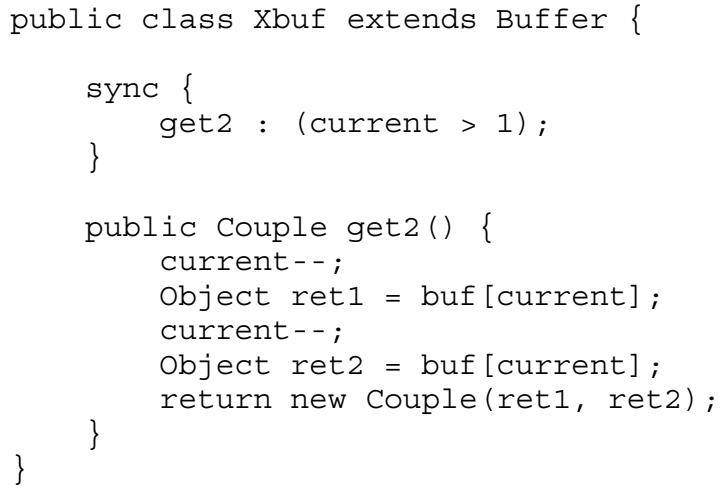

Figure 10. Inheriting synchronization constraints.

In Figure 8 we show an example of a class which does not override the bodies of its get and put methods, but overrides their synchronization constraints making them stricter. In this case we say that the synchronization constraint for the super-class has been covariantly redefined. In [9], the author favors this manner of synchronization overriding. There is, however, no general agreement on this issue. As an example the language Rosette [10] is based on making synchronization constraints less strict in the derived classes, and other authors argue in favor of this choice [11,12]. In Jeeg both manners of synchronization overriding are possible, indeed we believe that both techniques have their use in different situations. As an example of a derived class which makes the synchronization constraints of the parent less stringent, consider the simple class representing a resource (Figure 11). The base class Resource allows the acquire method to be called only when the resource is not already taken. The derived class ReadonlyResource must adopt a less stringent policy, it models a readonly resource, as a consequence it can be shared without mutual exclusion problems. For this reason it makes sense to allow multiple clients to share the resource, to accomplish this the synchronization constraint of the method acquire is made less stringent than in the parent class.

In Figure 12 we see the other extreme, a class which overrides a method but does not override its synchronization constraint which remains the one inherited. Its semantics are straightforward, the method get returns the object stored in the buffer as a chunk of bytes. Clearly this does not affect its concurrent behavior and it is safe to keep its synchronization constraint unchanged.

\section{Jeeg and exceptions}

Method execution might be stopped by the occurrence of a unhandled exception. With respect to the object history two possibilities arise. We could choose to keep the method in the history or ignore it. It is possible to provide examples favoring one or the other approach. Both solutions pose no 


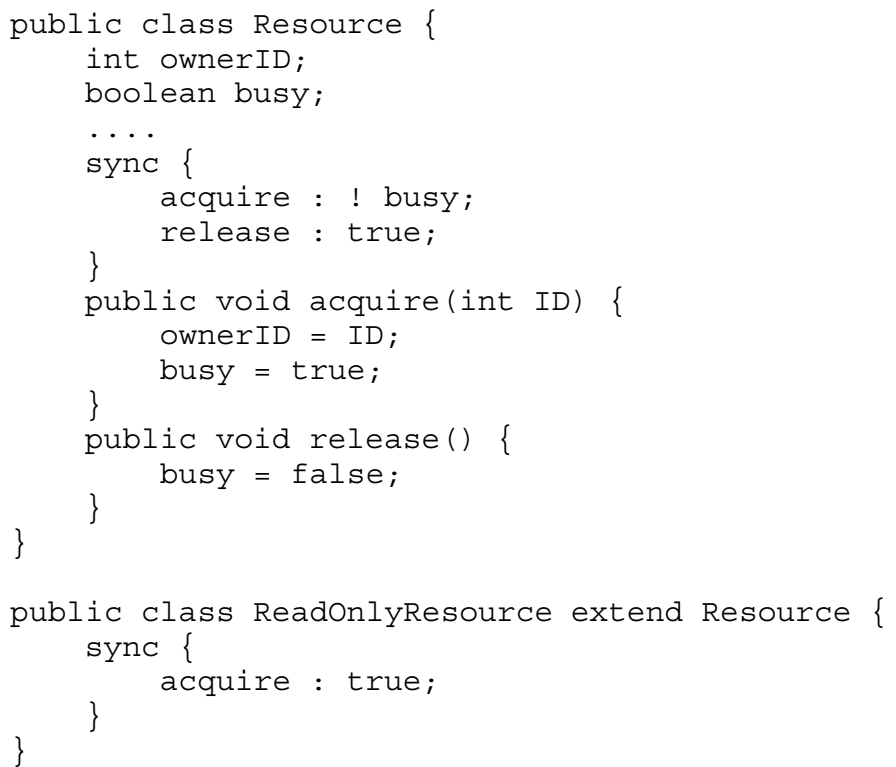

Figure 11. A resource hierarchy.

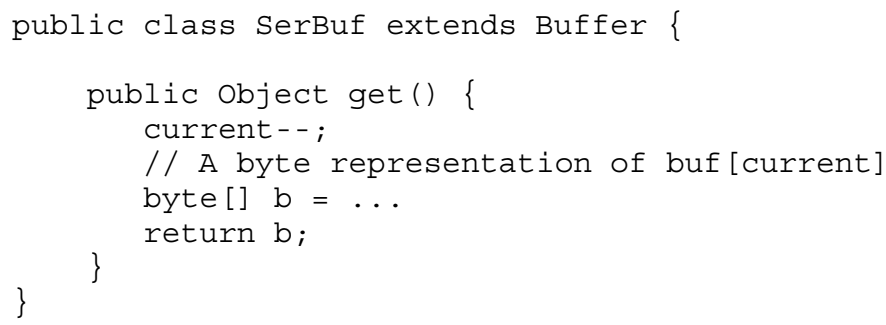

Figure 12. A serializing buffer. 
implementation challenges. In the current implementation, we chose to only put methods in the history which completed their execution.

\section{XML constraints}

In order to favor the separation between method definitions and synchronization code, Jeeg allows for the synchronization constraints to be specified separately in an XML file. When the Jeeg compiler processes a source file ClassName.j1 it looks for a XML file named ClassName.xml. If it finds the file then it validates it against the relevant DTD, which can be found in Appendix A. If the validation is successful the synchronization constraints it describes are weaved into the resulting class file. If a sync section is present in the class definition, it is overridden by the external constraints in the XML file.

To give a quick taste of how to define synchronization constraints using a XML file, consider the bounded buffer example in Figure 7. Its sync section is equivalent to the following XML description:

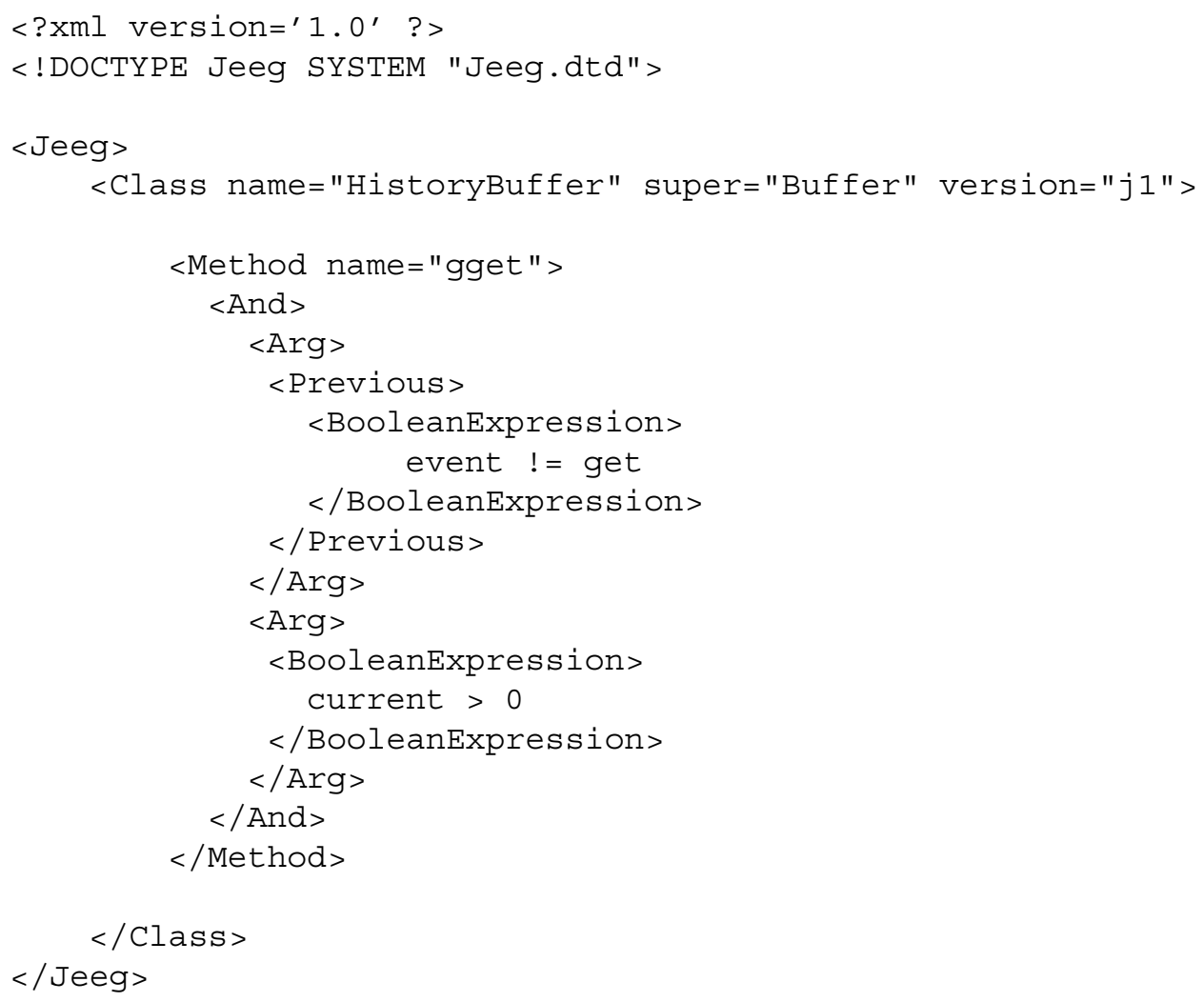




\section{IMPLEMENTATION}

The current Jeeg implementation $\$$ is a pre-processor which, given a Jeeg source file, generates an equivalent .java file and compiles it to bytecode. The resulting class files rely on a runtime system. The purpose of the runtime system is to implement a runtime evaluator for the $\mathrm{CL}$ formulae used in the program.

\section{Runtime evaluation of CL expressions}

The CL language is essentially a variation of LTL based on past-tense temporal operators. Every time a guarded method is called its execution depends on the truth value of a certain temporal formula: its synchronization constraint. If the constraint evaluates to true the method is executed, otherwise it is blocked until the condition becomes true.

Runtime evaluation of LTL formulae is a recurrent problem. In an wider context the problem can be stated as follows:

\section{Given a finite trace $\Sigma$ and a LTL formula $\phi$, does $\Sigma \models \phi$ ?}

This problem appears frequently when trying to apply model-checking techniques to the verification of Java or C++ programs [13-18].

Traditionally, LTL model checking is accomplished by first translating the LTL formula in a Büchi automata [19] and then proving properties on them [19,20]. Although in [13,14] the authors discuss why such a solution is not ideal to the runtime verification on finite traces, this approach is nevertheless used by the JPaX runtime analysis tool [15].

Dealing with past-tense operators gives us an advantage. The dynamic programming algorithm presented in [14] requires as input the trace of the program to evaluate a certain formula, indeed it traverses the program trace backwards. This implies that the algorithm is not 'online', i.e. it cannot be executed at the same time as the program it refers to. By duality, the same algorithm becomes online for the past fragment [21]. The algorithm has complexity $O(m)$, where $m$ is the size of the LTL formula. An alternative approach would rely on modifying the automata-based algorithm proposed in [15] to adapt them to past-tense operators.

An implementation has thus at least two choices available. The current Jeeg implementation relies on a variation of the dynamic programming algorithm. We found this choice to be the most natural. The algorithm is efficient, indeed weakening the logic would not result in a faster algorithm. Intuitively, given a Jeeg program and its set of synchronization constraints the compiler generates a runtime evaluation algorithm for them and weaves it into the business code of the program. At every step in the object history, i.e. method execution, the evaluator updates the truth values of the synchronization constraints.

The evaluation algorithm consists of (repeated) visits to the syntax tree of the formula.

To focus the ideas, let us consider the example of the temporal formula

$$
\text { (Previous }(x>0)) \& \& !(y<0)
$$

\footnotetext{
¥Available at: http://www.brics.dk/ milicia/Jeeg/.
} 
and its corresponding tree:

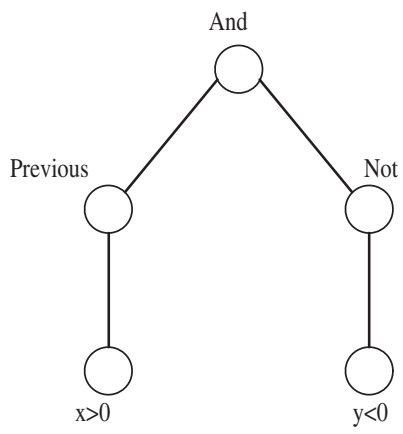

Every node of the tree represents a subformula of the original temporal formula and is labeled by two attributes, now and before, which hold the truth value of the corresponding sub-formula at the current time and one step before, respectively. The task of the algorithm is to visit the tree and update the values of the two attributes for every node.

In Section 2.1 we adopted strong semantics for our temporal operators; that is, we assumed that Previous can only be applied at times greater than zero. As a consequence, at the initial instant the before attribute of every sub-formula is set to false. The truth value of the now attribute is initialized when the object is created and depends on the initial state of the object. For every node $\phi$ we use the notation $\phi_{0}$ to refer to its first (left-wise) child and $\phi_{1}$ to its second child, if the node represents a binary operator. Attributes of the children are denoted by $\phi_{i}$.now and $\phi_{i}$.before. The algorithm performs a simple depth-first visit of the tree and for every node $\phi$ updates the value of the before and now fields. First we perform the assignment $\phi$.before $=\phi$.now, then, depending on the node type, we update the now field according to the following rules:

$\begin{array}{ll}\text { previous } & \text { now }=\phi_{0} . \text { before } \\ \text { always } & \text { now }=\text { before } \text { and } \phi_{0} \text {.now } \\ \text { sometimes } & \text { now }=\text { before } \text { or } \phi_{0} . \text { now } \\ \text { since } & \text { now }=\phi_{1} \text {.now } \text { or }\left(\text { before } \text { and } \phi_{0} . \text { now }\right) \\ \text { and } & \text { now }=\phi_{0} \text {.now } \text { and } \phi_{1} \text {.now } \\ \text { or } & \text { now }=\phi_{0} \text {.now } \text { or } \phi_{1} \text {.now } \\ \text { not } & \text { now }=\operatorname{not} \phi_{0} \text {.now } \\ \text { AP } & \text { now }=\operatorname{eval}(\phi)\end{array}$

To clarify the working of the algorithm, consider a simple formula

$\operatorname{Previous}(x==1)$

and a trivial counter class such as that we presented in Figure 1. Suppose we execute the code:

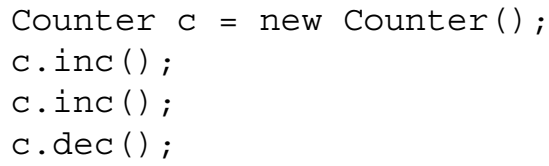




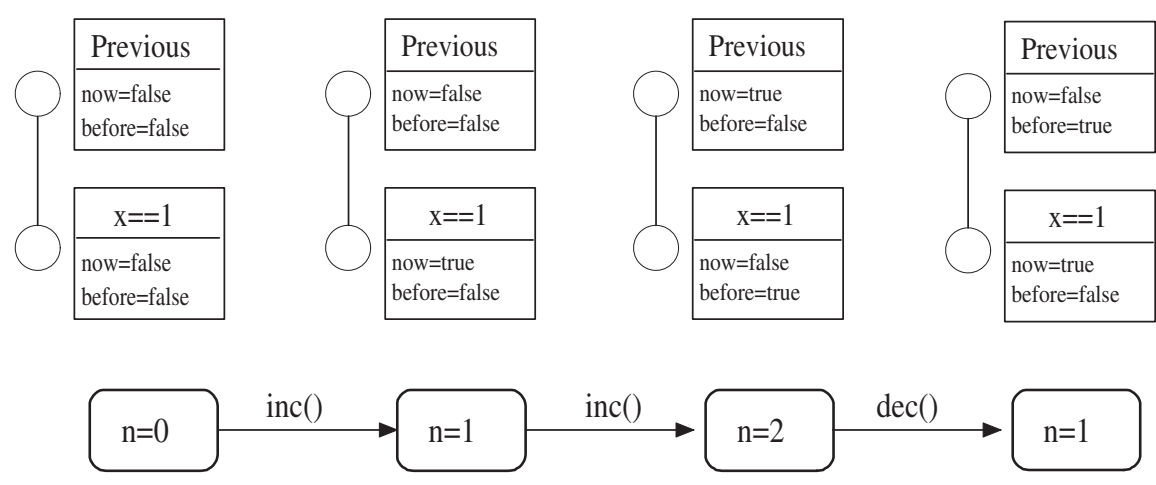

Figure 13. History.

then Figure 13 shows how the attributes in the formula tree evolve with respect to the history of the object $\mathrm{C}$.

It is easy to see that the complexity of the runtime evaluation algorithm is linear in the size of the formula tree. The runtime overhead involved is thus linear in the size of the synchronization constraints.

\section{Synchronization manager}

For the evaluation algorithm to be sound, formulae must be evaluated at every step in the program history, i.e. after every method execution. This is accomplished by a synchronization manager through a mechanism of method call interception (MCI), typical of the implementation of aspect-oriented languages.

The synchronization manager takes control after a method call. Then it checks whether the synchronization constraint for the method is verified. Note that the constraint must not be evaluated at this stage, its truth value is already available. This is the case as the truth value of synchronization constraints is updated after the execution of a method. If the constraint is true the control goes back to the method code, otherwise the synchronization manager performs a wait ( ) and puts the method on hold. After the execution of a method is accomplished, the control shifts back to the synchronization manager. At this point the synchronization constraints are evaluated. Since the execution of a method may change the state of the object, after updating the value of the synchronization constraints the manager issues a notifyAll () statement. Blocked methods may then attempt to proceed again.

To perform its tasks the synchronization manager must have access to the private/protected fields of the object. To accomplish this we chose to make the synchronization manager an inner class of the object it manages.

A complete example showing the Java code generated from a Jeeg source file can be found in Appendix B. 


\section{BENCHMARKS}

To assess the feasibility of our approach we performed some targeted benchmarking on the current prototype implementation of the Jeeg compiler. In this section we outline our results.

\subsection{General setting}

When benchmarking code running in a JVM care must be taken to avoid interference from the garbage collector. Furthermore a single measurement is no valid indication of the actual time spent during an operation. Multiple measurement of the same experiment must be performed instead. We take their average as a fair result of our experiment.

Although Java is designed to be platform independent, different implementations of the virtual machine for different operating systems might perform differently. We chose to perform our tests on two popular operating systems: Linux and Windows 2000.

We chose to run the virtual machine with no optimizations, in particular the code was only interpreted, the just-in-time compiler was turned off. In this manner we could run the same tests a number of times without speed-ups. Our benchmarks are thus a measure of the worst case scenario, when the code is executed only once and thus no gain is to be expected by just-in-time compilation. All the programs were compiled and run using the J2SE 1.4 and the -Xint option.

To have a better feel of the performance impact in a realistic setting we performed our tests on low-end and high-end machines. Below we list the machines we used.

- Machine 1: AMD 1800+XP, 256 MB, Windows 2000, Jdk 1.4.

- Machine 2: AMD 1800+XP, 256 MB, Linux RedHat 6.2, Jdk 1.4.

- Machine 3: Celeron 300 Mhz, 192 MB, Windows 2000, Jdk 1.4.

- Machine 4: Pentium 4 1.6 Ghz, 512 MB, Linux 2.4.18, Jdk 1.4.

The code used for the benchmarks is available on the Web at http://www.brics.dk/ milicia/Jeeg.

\subsection{Benchmark results}

The overhead introduced by our methodology is felt first at the time of object creation, and then whenever a call to a synchronized method is performed. We begin by showing the test results in these two situations and conclude with an evaluation of the performance impact of the Jeeg methodology.

\section{Object creation}

At object creation time the structures representing the (temporal) formulae of the synchronization constraints must be built. This results in the creation of as many objects as logic operators present in the formulae. As a consequence we expect object creation to become slower as synchronization constraints grow more complex. To quantify the overhead we timed the creation of objects with increasing complex synchronization constraints (in the size of the formulae involved). The constructor of the object was otherwise empty. The results of our tests can be found in Figure 14. 

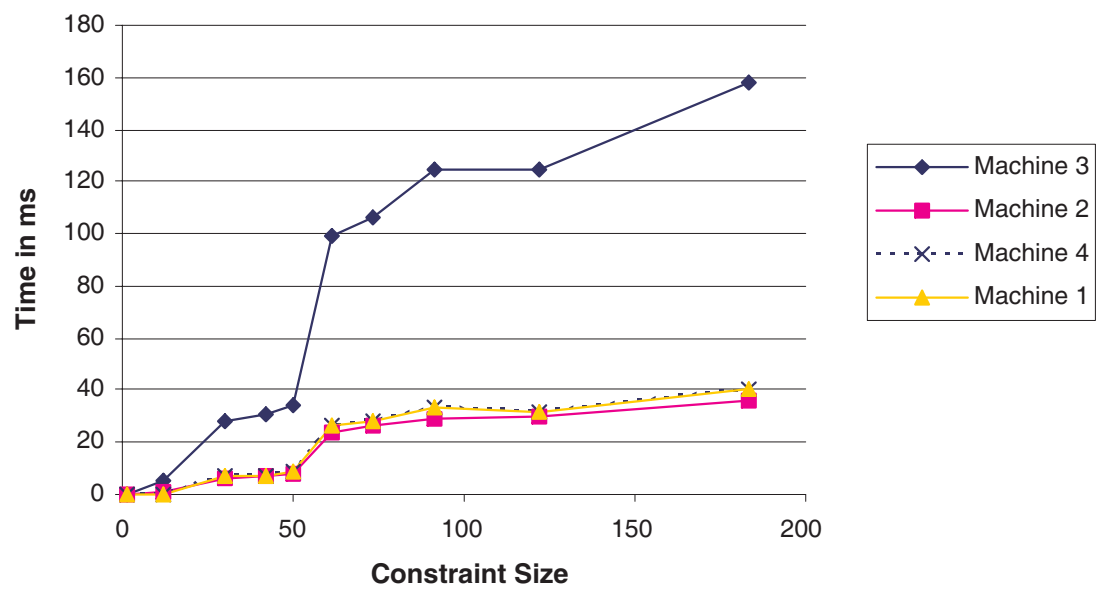

Figure 14. Object creation overhead.

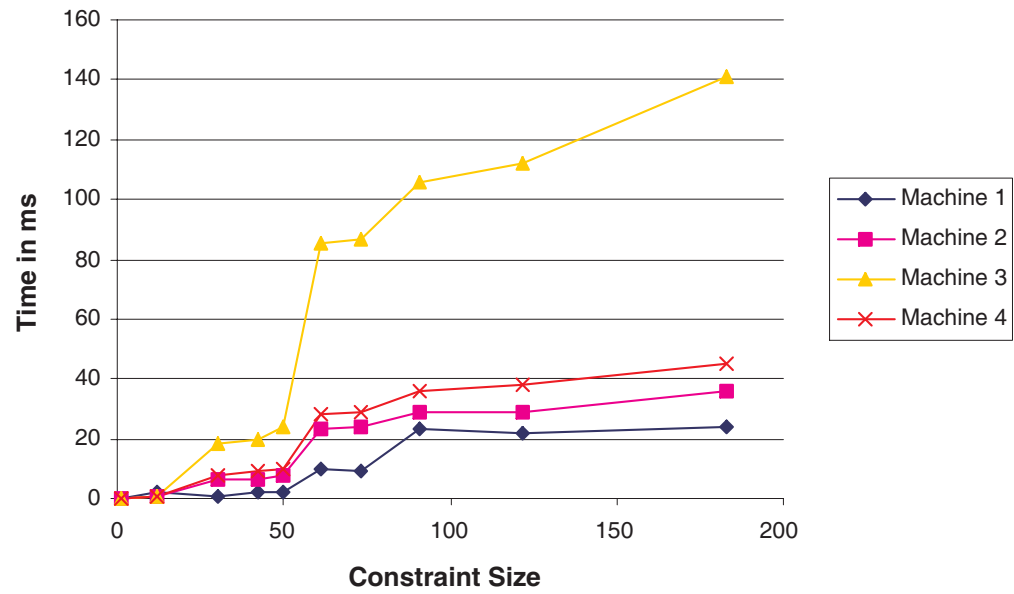

Figure 15. Method call overhead. 

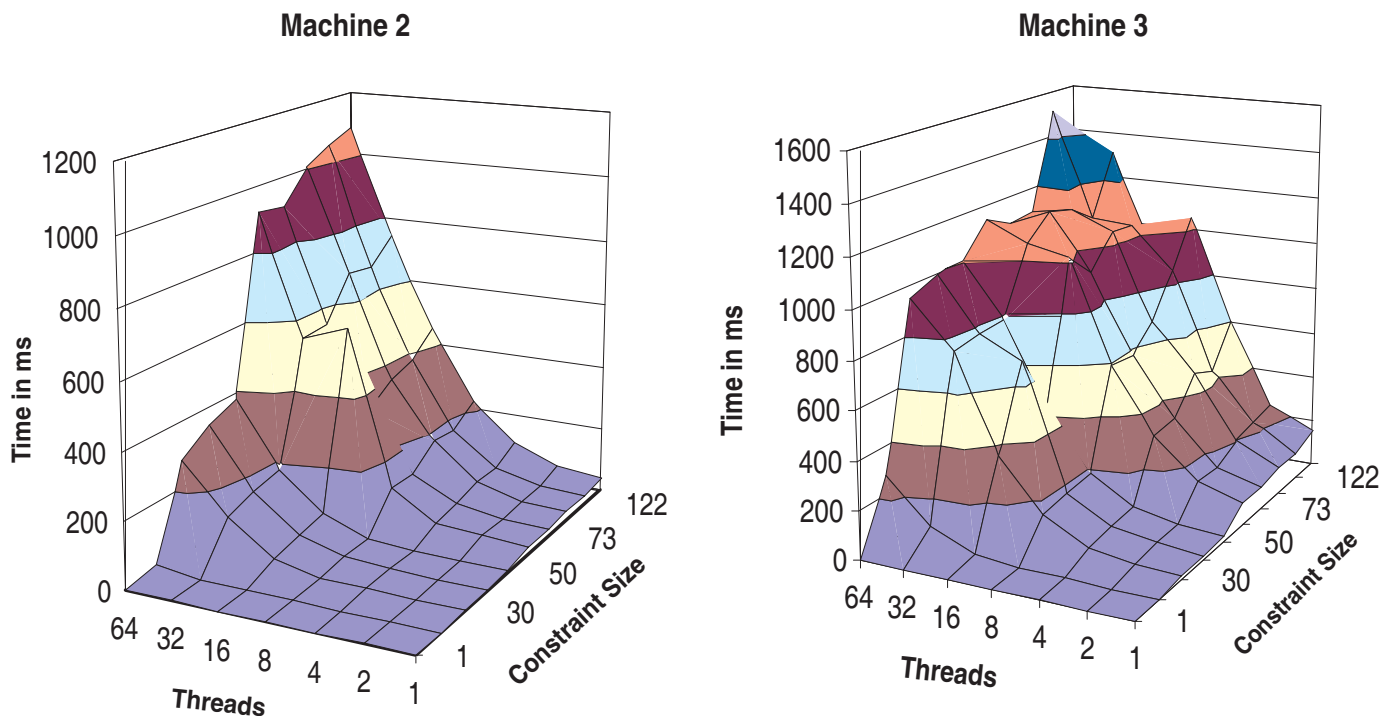

Figure 16. Method call overhead.

\section{Method call}

Every time a (synchronized) method is called the algorithm described in Section 6 must be performed. This results in the evaluation of all synchronization constraints. The overhead we face is thus proportional to the sum of the sizes of the logic formulae describing the constraints. Clearly every method call will incur the same overhead regardless of the size of its own synchronization constraint.

To measure the overhead involved in our technique we tested method calls on objects with increasing complex synchronization constraints. We made sure, to avoid any biased result, that the constraints would always evaluate to true. Method calls performed no function, in this way we made sure that we only measured the unavoidable overhead brought up by our technique. The results of our tests can be seen in Figure 15.

A different performance problem could result from the fact that the synchronization constraints must be evaluated in mutual exclusion. The object will be locked during the evaluation. If a number of threads are actively accessing the object this could slow down the method calls sensibly. To evaluate this issue we performed the test above with an increasing number of threads. The results can be found in Figure 16. We can see that in the presence of large constraints and over 50 threads actively using the objects we face a sensible slow-down.

We wish to remark that Jeeg takes care of all the synchronization constraints of the object. An equivalent Java program must accomplish the same results in a different fashion, for example using Boolean variables to keep track of its state. An interesting experiment is thus the comparison of 


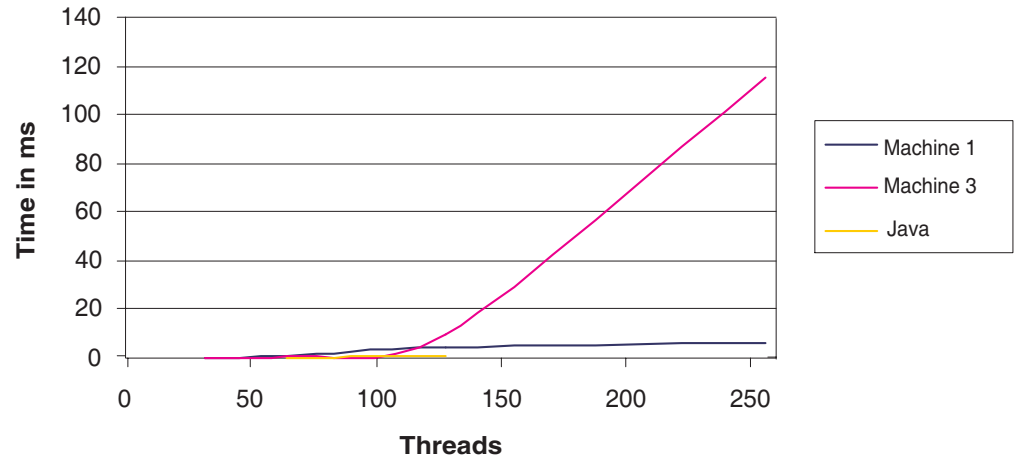

Figure 17. HistoryBuffer performances.

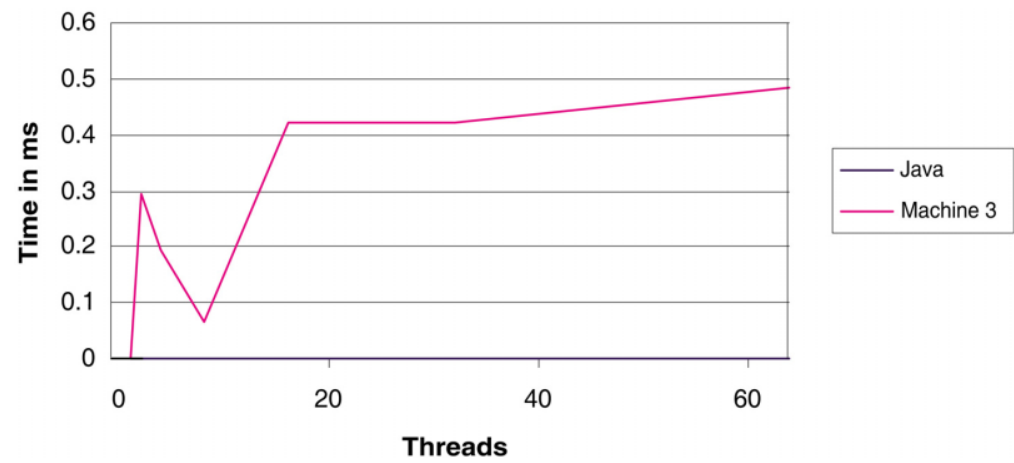

Figure 18. HistoryBuffer performances.

two semantically equivalent Jeeg and Java programs. We use as our test-bed the HistoryBuffer example of Section 3. Figure 17 compares the execution time for a method call to a Java implementation of the class HistoryBuffer (as seen in Figure 5) and its Jeeg counterpart (as seen in Figure 7). The high-end machines feel almost no performance loss; on the other hand, if many threads are active at the same time, the low-end machine suffers from severe performance losses. However, even the low-end machine performs well in the presence of as many as 64 active threads, as Figure 18 shows.

\subsection{Evaluation}

Our tests show that under low-load (below 70 threads) even the most complex synchronization constraints yield little performance overhead. Low-end machines face worse scalability problems due 
to the additional time the object is kept locked. If the machine cannot perform the evaluation algorithm fast enough a number of threads will be kept waiting.

Experience shows that the synchronization constraints of an object seldom reach a length of over 10 or 20 logical connectives. Our benchmarks show that for such objects the performance loss is negligible even in case of high-load (more than 200 active threads).

We are currently evaluating possible optimization strategies for the formulae evaluation algorithm.

\section{RELATED WORK}

The idea of specifying synchronization constraints in programming (as opposed to verifying) using a temporal logic has, to the best of our knowledge, not been explored before. Indeed, the problem of runtime evaluation of LTL formulae has only recently come to the attention of the research community [13,17].

The idea of a complete separation between the definition of a method and its synchronization constraints is known to be helpful in avoiding the inheritance anomaly [3,22,23]. In this work, we uphold the concept by making synchronization code and method definitions totally independent, to the degree that they do not need to be specified in the same file. In this regard Jeeg is inspired by the current trends in component-based and aspect-oriented programming [24].

Frølund proposed a methodology for selective inheritance of synchronization constraints [9]. His proposal, based on method guards, favors the covariant redefinition of synchronization constraints in derived classes. As we pointed out in Section 5, this manner of synchronization redefinition is not universally accepted. Indeed, some languages $[10,25]$ take the opposite view and allow the derived class to make the synchronization constraints less stringent, that is contravariant. Examples exist in favor of both approaches; as a consequence we decided to allow both manners of overriding. From the point of view of the inheritance anomaly, Frølund's methodology is subject to the usual problems related to method guards, i.e. the history dependent variants of the anomaly.

Meseguer [25], analyzed the problem of the inheritance anomaly in the context of his rewriting logic-based language Maude [26]. Meseguer's work aimed at removing the need for synchronization code in the first place. This technique, based on rewriting logic, is closely tied to the Maude system and we are not aware of any adaption to imperative object-oriented languages such as Java.

Different lines of work were taken by Matsuoka and Yonezawa: the first based on the notion of reflection [3], the second aiming at reducing the amount of synchronization code to a minimum [3].

An approach more in line with aspect-oriented programming is presented in [27]. Although their use of abstract communication types (ACTs) does provide a way to tackle the history sensitive anomaly in a modular fashion, it is still based on ad hoc coding. Every instance of the anomaly requires the programmer to write a specific ACT to solve it. The problem is thus moved from the object to the ACT rather than solved. Similar results were obtained using the synchronization patterns [23] and synchronization rings [28] methodologies.

\section{CONCLUSIONS}

We introduced Jeeg, a dialect of Java where synchronization constraints are written in LTL and are specified in a declarative manner. We showed by examples that the additional expressive power of 
our synchronization language, $\mathrm{CL}$, is helpful in treating the inheritance anomaly. Also, we provided a characterization of the expressiveness of $\mathrm{CL}$ in terms of regular languages that yields a precise description of the sequences of events we can express. Finally, we described the current implementation of Jeeg.

Propositional LTL seems to us to offer an excellent balance between expressiveness and computational overhead. It would indeed be interesting to base Jeeg on quantified linear temporal logic (QLTL) or monadic second-order logic (MSOL), 'second-order' variations of LTL of greater expressiveness. In particular, QLTL and MSOL correspond to regular languages in the same sense as LTL corresponds to star-free regular languages. However, while giving us the power to express synchronization policies as complex as regular languages or more, these options would present an increased computational cost that we are currently investigating.

With regards to the Jeeg compiler, we are exploring the possibility of optimizing the LTL evaluation procedure by using ad hoc static-analysis techniques.

The current implementation of the Jeeg compiler is available at http://www.brics.dk/ milicia/Jeeg/.

\section{APPENDIX A. THE JEEG DTD}

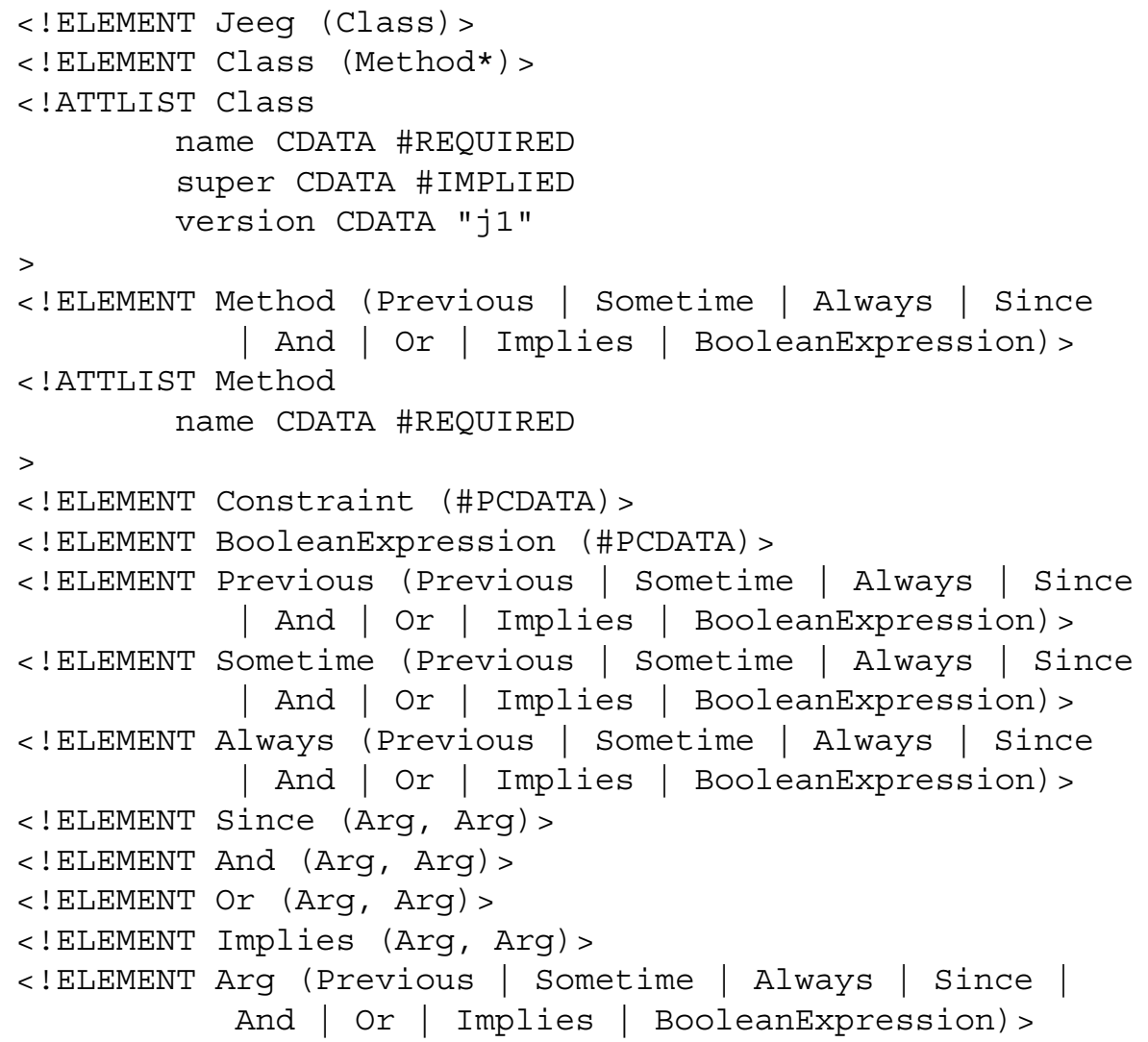




\section{APPENDIX B. THE JAVA CODE GENERATED FROM THE BUFFER EXAMPLE}

In this appendix we show the code generated by the Jeeg compiler from the Buffer example presented in Section 3. Interface and class names referring to the Jeeg runtime system are normally fully qualified. However, to keep names short here we write, for instance, Propositional Formula instead of the fully qualified org.brics.gm.jeeg. formulae. PropositionalFormula.

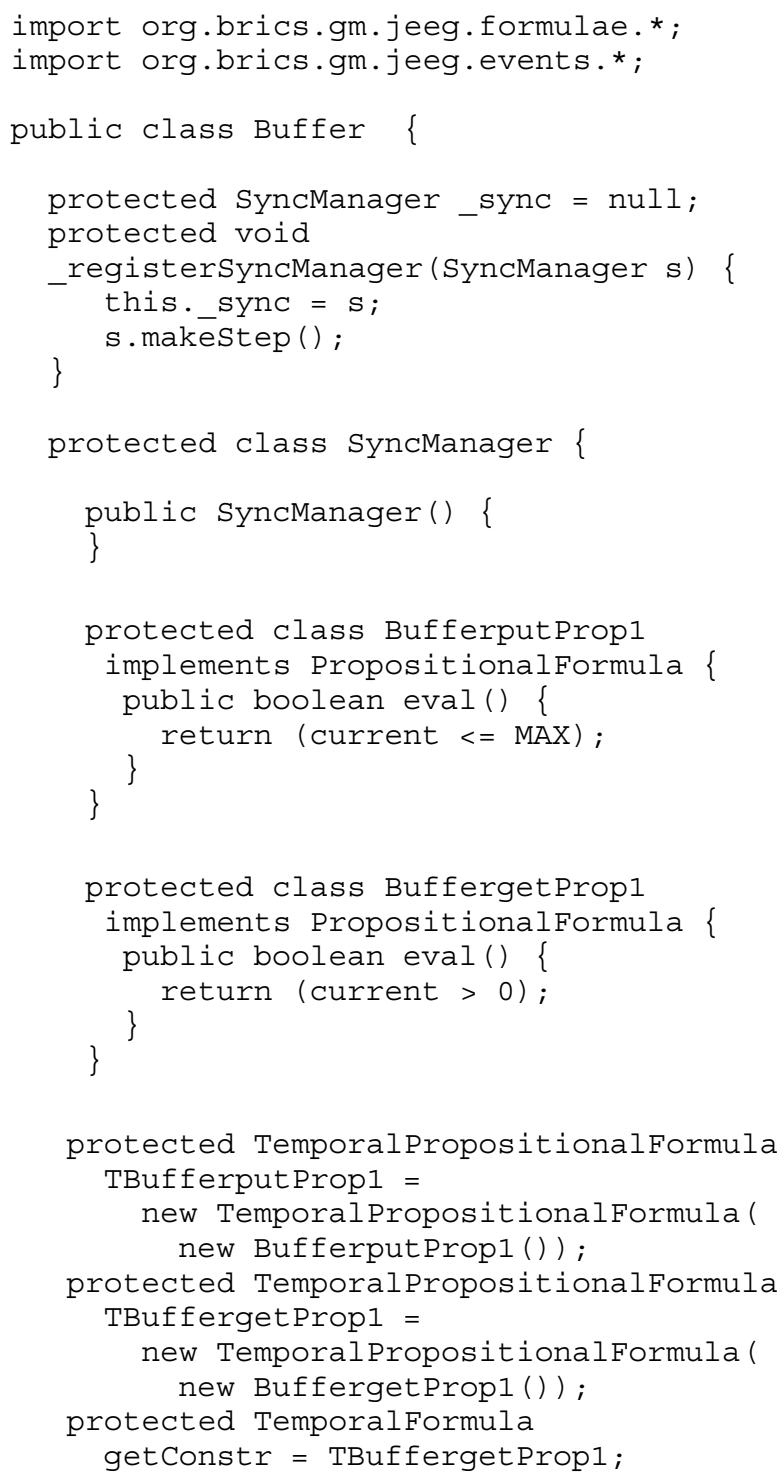

The include statements above refer the Jeeg runtime system.

Every Jeeg class requires a synchronization manager as described in Section 6. The method registersyncmanager is used at object creation time to specify which synchronization manager will take care of the class.

The synchronization manager is inserted as an inner class.

Propositional formulae ( $p \in \mathrm{AP}$ ), are wrapped into classes. Observe that the Java inner class mechanism grants the synchronization manager full access on the private/protected attributes of the surrounding class.

Appropriate temporal formulae representing the synchronization constraints of the object are instantiated. The classes used are taken from the Jeeg runtime system. 


\section{REFERENCES}

1. America P. POOL: Design and experience. OOPS Messenger 1991; 2(2):16-20.

2. Briot J-P, Yonezawa A. Inheritance and synchronization in concurrent OOP. Proceedings of the European Conference on Object-Oriented Programming (ECOOP'87) (Lecture Notes in Computer Science, vol. 276). Springer: Berlin, 1987; $32-40$.

3. Matsuoka S, Yonezawa A. Analysis of inheritance anomaly in object-oriented concurrent programming language. Research Directions in Concurrent Object-Oriented Programming, Gul A, Wegner P, Akinori Y (eds.). MIT Press: Cambridge, MA, 1993; 107-150.

4. Pnueli A. The temporal logic of programs. Proceedings of the 18th IEEE Symposium on the Foundations of Computer Science (FOCS-77). IEEE Computer Society Press: Los Alamitos, CA, 1977; 46-57.

5. Lichtenstein O, Pnueli A, Zuck L. The glory of the past. Proceedings of the 3rd Workshop on Logics of Programs, Brooklyn, NY, 17-19 June 1985 (Lecture Notes in Computer Science, vol. 193), Parikh R, (ed.). Springer: Berlin, 1985; 196-218.

6. Emerson EA. Temporal and Modal Logic (Handbook of Theoretical Computer Science, vol. B), van Leeuwen J (ed.). Elsevier: Amsterdam, 1990; 996-1072.

7. Zuck L. Past temporal logic. PhD Thesis, Weizmann Institute, 1986.

8. Wolfgang T. Languages, Automata and Logic (Handbook of Theoretical Computer Science, vol. B), van Leeuwen J (ed.). Elsevier: Amsterdam, 1990.

9. Frølund S. Inheritance of synchronization constraints in concurrent object-oriented programming languages. European Conference on Object-Oriented Programming (ECOOP'92) (Lecture Notes in Computer Science, vol. 615). Springer: Berlin, 1992; 185-196.

10. Tomlinson C, Singh V. Inheritance and synchronization with enabled-sets. Proceedings of the Conference on Objectoriented Programming Systems, Languages and Applications (OOPSLA'99). ACM Press: New York, 1989.

11. Nierstrasz O, Papathomas M. Towards a type theory for active objects. Proceedings of the Workshop on Object-Based Concurrent Systems (OOPSLA/ECOOP90). ACM OOPS Messenger 1991; 2(2):89-93.

12. Nierstrasz O, Papathomas M. Viewing objects as patterns of communicating agents. Proceedings of the Conference on Object-oriented Programming Systems (OOPSLA/ECOOP'90), Languages and Applications. ACM SIGPLAN Notices 1990; 25(10):38-43.

13. Havelund K, Rosu G. Testing linear temporal logic formulae on finite execution traces. Technical Report TR 01-08, RIACS, May 2001.

14. Rosu G, Havelund K. Synthesizing dynamic programming algorithms from linear temporal logic formulae. Technical Report TR 01-15, RIACS, May 2001.

15. Giannakopoulou D, Havelund K. Automata-based verification of temporal properties on running programs. Automated Software Engineering 2001 (ASE'01), San Diego, CA, November 2001. IEEE Computer Society Press: Los Alamitos, CA, 2001.

16. Havelund K, Rosu G. Monitoring programs using rewriting. Automated Software Engineering 2001 (ASE'01), San Diego, CA, November 2001. IEEE Computer Society Press: Los Alamitos, CA, 2001.

17. Drusinsky D. The temporal rover and the ATG rover. SPIN Model Checking and Software Verification (Lecture Notes in Computer Science, vol. 1885). Springer: Berlin, 2000; 323-330.

18. Lee I, Kannan S, Kim M, Sokolsky O, Viswanathan M. Runtime assurance based on formal specifications. Proceedings of the International Conference on Parallel and Distributed Processing Techniques and Applications. CSREA Press: Las Vegas, NV, 1999.

19. Clarke EM, Grumberg O, Peled SA. Model Checking. MIT Press: Cambridge, MA, 1999.

20. Holzmann GJ. The model checker SPIN. IEEE Transactions on Software Engineering (Special Issue on Formal Methods in Software Practice) 1997; 23(5):279-295.

21. Havelund K, Rosu G. Monitoring Java programs with Java Pathexplorer. First Workshop on Runtime Verification (RV'01) (Electronic Notes in Theoretical Computer Science, vol. 55). Elsevier: Amsterdam, 2001.

22. Matsuoka S, Wakita K, Yonezawa A. Synchronization constraints with inheritance: What is not possible-so what is? Technical Report TR 90-10, Department of Information Science, The University of Tokyo, 1989.

23. Videira Lopes C, Lieberherr KJ. Abstracting Process-to-function Relations in Concurrent Object-oriented Applications (Lecture Notes in Computer Science, vol. 821). Springer: Berlin, 1994; 81-99.

24. Kiczales G, Lamping J, Mendhekar A, Maeda C, Lopes C, Loingtier J-M, Irwin J. Aspect-oriented programming. Object-Oriented Programming (ECOOP'97) (Lecture Notes in Computer Science, vol. 1241). Springer: Berlin, 1997; 220-242.

25. Meseguer J. Solving the inheritance anomaly in concurrent object-oriented programming. Proceedings of the European Conference on Object-oriented Programming (ECOOP'93) (Lecture Notes in Computer Science, vol. 707). Springer: Berlin, 1993; 220-246. 
26. Meseguer J, Winkier T. Parallel programming in MAUDE. Proceedings of Research Directions in High-Level Parallel Programming Languages (Lecture Notes in Computer Science, vol. 574). Springer: Berlin, 1992; 253-295.

27. Bergmans L. Composing concurrent objects. PhD Thesis, University of Twente, 1994.

28. Holmes D. Synchronization rings—composable synchronization for object-oriented systems. PhD Thesis, Macquarie University, 1999 